\title{
Complexity of Products of Some Complete and Complete Bipartite Graphs
}

\author{
S. N. Daoud ${ }^{1,2}$ \\ ${ }^{1}$ Department of of Mathematics, Faculty of Science, Taibah University, Al Madinah 344, Saudi Arabia \\ ${ }^{2}$ Department of Mathematics, Faculty of Science, El-Menoufia University, Shebeen El-Kom, Egypt
}

Correspondence should be addressed to S. N. Daoud; salamadaoud@gmail.com

Received 2 June 2013; Revised 29 July 2013; Accepted 12 August 2013

Academic Editor: Roberto Barrio

Copyright (c) 2013 S. N. Daoud. This is an open access article distributed under the Creative Commons Attribution License, which permits unrestricted use, distribution, and reproduction in any medium, provided the original work is properly cited.

\begin{abstract}
The number of spanning trees in graphs (networks) is an important invariant; it is also an important measure of reliability of a network. In this paper, we derive simple formulas of the complexity, number of spanning trees, of products of some complete and complete bipartite graphs such as cartesian product, normal product, composition product, tensor product, and symmetric product, using linear algebra and matrix analysis techniques.
\end{abstract}

\section{Introduction}

In this work we deal with simple and finite undirected graphs $G=(V, E)$, where $V$ is the vertex set and $E$ is the edge set. For a graph $G$, a spanning tree in $G$ is a tree which has the same vertex set as $G$. The number of spanning trees in $G$, also called the complexity of the graph, denoted by $\tau(G)$, is a wellstudied quantity (for long time). A classical result of Kirchhoff [1], can be used to determine the number of spanning trees for $G=(V, E)$. Let $V=\left\{v_{1}, v_{2}, \ldots, v_{n}\right\}$; then the Kirchhoff matrix $H$ defined as $n \times n$, characteristic matrix, $H=D-A$, where $D$ is the diagonal matrix whose elements are the degrees of the vertices of $G$. While $A$ is the adjacency matrix of $G, H=\left[a_{i j}\right]$ is defined as follows:

(i) $a_{i j}=-1 v_{i}$ and $v_{j}$ are adjacent and $i \neq j$,

(ii) $a_{i j}$ equals the degree of vertex $v_{i}$ if $i=j$,

(iii) $a_{i j}=0$ otherwise.

All of the cofactors of $H$ are equal to $\tau(G)$. There are other methods for calculating $\tau(G)$. Let $\mu_{1} \geq \mu_{1} \geq \cdots \geq \mu_{p}$ denote the eigenvalues of $H$ matrix of a $p$ point graph. Then it is easily shown that $\mu_{p}=0$. Furthermore, Kelmans and Chelnokov [2] have shown that $\tau(G)=(1 / p) \prod_{k=1}^{p-1} \mu_{k}$. The formula for the number of spanning trees in a d-regular graph $G$ can be expressed as $\tau(G)=(1 / p) \prod_{k=1}^{p-1}\left(d-\lambda_{k}\right)$, where $\lambda_{0}=d, \lambda_{1}, \lambda_{2}, \ldots, \lambda_{p-1}$ are the eigenvalues of the corresponding adjacency matrix of the graph. However, for a few special families of graphs there exist simple formulas that make it much easier to calculate and determine the number of corresponding spanning trees especially when these numbers are very large. One of the first results is due to Cayley [3] who showed that the complete graph on $n$ vertices, $K_{n}$ has $n^{n-2}$ spanning trees, $n \geq 2$. Another result is that $\tau\left(K_{p, q}\right)=$ $p^{q-1} q^{p-1}, p, q \geq 1$, where $K_{p, q}$ is the complete bipartite graph with bipartite sets containing $p$ and $q$ vertices, respectively. It is well known, as in, for example, $[4,5]$. Another result is due to Sedláček [6] who derived a formula for the wheel on $n+1$ vertices, $W_{n+1}$; he showed that $\tau\left(W_{n+1}\right)=((3+\sqrt{5}) / 2)^{n}+$ $((3-\sqrt{5}) / 2)^{n}-2$, for $n \geq 3$. Sedlacek [7] also later derived a formula for the number of spanning trees in a Mobius ladder, $M_{n}, \tau\left(M_{n}\right)=(n / 2)\left[(2+\sqrt{3})^{n}+(2-\sqrt{3})^{n}+2\right]$ for $n \geq 2$. Another class of graphs by Boesch et al., for which an explicit formula has been derived, is based on a prism $[8,9]$.

Now, we can introduce the following lemmas.

Lemma 1 (see [10]). Consider $\tau(G)=\left(1 / n^{2}\right) \operatorname{det}(n I-\bar{D}+\bar{A})$ where $\bar{A}$ and $\bar{D}$ are the adjacency and degree matrices of $\bar{G}$ and the complement of $G$, respectively, and $I$ is the $n \times n$ unit matrix. 
Lemma 2. Let $E_{n}(x)$ be $n \times n$ matrix, $x \geq 2$ such that

$$
E_{n}(x)=\left(\begin{array}{cccccc}
x & 1 & \cdots & \cdots & \cdots & 1 \\
1 & \ddots & \ddots & \ddots & \ddots & \vdots \\
\vdots & \ddots & \ddots & \ddots & \ddots & \vdots \\
\vdots & \ddots & \ddots & \ddots & \ddots & \vdots \\
\vdots & \ddots & \ddots & \ddots & \ddots & 1 \\
1 & \cdots & \cdots & \cdots & 1 & x
\end{array}\right)
$$

Then,

$$
\operatorname{det}\left(E_{n}\right)=(x+n-1)(x-1)^{n-1} .
$$

Proof. From the definition of the circulant determinants, we have

$$
\begin{aligned}
\operatorname{det}\left(E_{n}(x)\right)= & \operatorname{det}\left(\begin{array}{cccccc}
x & 1 & \cdots & \cdots & \cdots & 1 \\
1 & \ddots & \ddots & \ddots & \ddots & \vdots \\
\vdots & \ddots & \ddots & \ddots & \ddots & \vdots \\
\vdots & \ddots & \ddots & \ddots & \ddots & \vdots \\
\vdots & \ddots & \ddots & \ddots & \ddots & 1 \\
1 & \cdots & \cdots & \cdots & 1 & x
\end{array}\right) \\
= & \prod_{j=1}^{n}\left(x+\omega_{j}+\omega_{j}^{2}+\omega_{j}^{3}+\cdots+\omega_{j}^{n-1}\right) \\
= & (x+1+1+\cdots+1) \\
& \times \prod_{j=1, \omega_{j} \neq 1}^{n}(x+\underbrace{\omega_{j}+\omega_{j}^{2}+\omega_{j}^{3}+\cdots+\omega_{j}^{n-1}}_{=-1}) \\
= & (x+n-1) \times(x-1)^{n-1} .
\end{aligned}
$$

We can generalize the previous lemma as follows.
Lemma 3. Let $A, B \in F^{n \times n}$ and $F \in F^{k n \times k n}$ such that

$$
F=\left(\begin{array}{cccccc}
A & B & \cdots & \cdots & \cdots & B \\
B & \ddots & \ddots & \ddots & \ddots & \vdots \\
\vdots & \ddots & \ddots & \ddots & \ddots & \vdots \\
\vdots & \ddots & \ddots & \ddots & \ddots & \vdots \\
\vdots & \ddots & \ddots & \ddots & \ddots & B \\
B & \cdots & \cdots & \cdots & B & A
\end{array}\right) .
$$

Then,

$$
\operatorname{det} F=[\operatorname{det}(A-B)]^{k-1} \operatorname{det}[A+(k-1) B] .
$$

Lemma 4 (see [11]). Let $A \in F^{n \times n}$, let $B \in F^{n \times m}$, let $C \in F^{m \times n}$, and let $D \in F^{m \times m}$; assume that $A, D$ are nonsingular matrices. Then

$$
\begin{aligned}
\operatorname{det}\left(\begin{array}{ll}
A & B \\
C & D
\end{array}\right) & =(-1)^{n m} \operatorname{det}\left(A-B D^{-1} C\right) \operatorname{det} D \\
& =(-1)^{n m} \operatorname{det} A \operatorname{det}\left(D-C A^{-1} B\right) .
\end{aligned}
$$

Formulas in Lemmas 2, 3, and 4 give some sort of symmetry in some matrices which facilitates our calculation of determinants.

\section{Number of Spanning Trees of Cartesian Product of Graphs}

The Cartesian product, $G_{1} \times G_{2}$, is the simple graph with vertex set $V\left(G_{1} \times G_{2}\right)=V_{1} \times V_{2}$ and edge set $E\left(G_{1} \times G_{2}\right)=$ $\left[\left(E_{1} \times V_{2}\right) \cup\left(V_{1} \times E_{2}\right)\right]$ such that two vertices $\left(u_{1}, u_{2}\right)$ and $\left(v_{1}, v_{2}\right)$ are adjacent in $G_{1} \times G_{2}$ if and only if either $u_{1}=v_{1}$ and $u_{2}$ is adjacent to $v_{2}$ in $G_{2}$ or $u_{1}$ is adjacent to $v_{1}$ in $G_{1}$ and $u_{2}=v_{2}[12]$.

Theorem 5. For $n, m \geq 1$, we have

$$
\begin{aligned}
\tau\left(K_{2} \times K_{m, n}\right)= & m^{n-1} n^{m-1}(m+2)^{n-1} \\
& \times(n+2)^{m-1}(n+m+2) .
\end{aligned}
$$

Proof. Applying Lemma 1, we have

$$
\begin{aligned}
& \tau\left(K_{2} \times K_{m, n}\right) \\
& =\frac{1}{(2(m+n))^{2}} \operatorname{det}(2(m+n) I-\bar{D}+\bar{A}) \\
& =\frac{1}{4(m+n)^{2}}
\end{aligned}
$$




$$
\begin{aligned}
& \times \operatorname{det}\left(\begin{array}{cccccccccccccccc}
n+2 & 1 & \cdots & 1 & 0 & \cdots & \cdots & 0 & 0 & 1 & \cdots & \cdots & \cdots & \cdots & \cdots & 1 \\
1 & \ddots & \ddots & \vdots & \vdots & \ddots & \ddots & \vdots & 1 & \ddots & \ddots & \ddots & \ddots & \ddots & \ddots & \vdots \\
\vdots & \ddots & \ddots & 1 & \vdots & \ddots & \ddots & \vdots & \vdots & \ddots & \ddots & \ddots & \ddots & \ddots & \ddots & \vdots \\
1 & \cdots & 1 & n+2 & 0 & \cdots & \cdots & 0 & \vdots & \ddots & \ddots & \ddots & \ddots & \ddots & \ddots & \vdots \\
0 & \cdots & \cdots & 0 & m+2 & 1 & \cdots & 1 & \vdots & \ddots & \ddots & \ddots & \ddots & \ddots & \ddots & \vdots \\
\vdots & \ddots & \ddots & \vdots & 1 & \ddots & \ddots & \vdots & \vdots & \ddots & \ddots & \ddots & \ddots & \ddots & \ddots & \vdots \\
\vdots & \ddots & \ddots & \vdots & \vdots & \ddots & \ddots & 1 & \vdots & \ddots & \ddots & \ddots & \ddots & \ddots & \ddots & 1 \\
0 & \cdots & \cdots & 0 & 1 & \cdots & 1 & m+2 & 1 & \cdots & \cdots & \cdots & \cdots & \cdots & 1 & 0 \\
0 & 1 & \cdots & \cdots & \cdots & \cdots & \cdots & 1 & n+2 & 1 & \cdots & 1 & 0 & \cdots & \cdots & 0 \\
1 & \ddots & \ddots & \ddots & \ddots & \ddots & \ddots & \vdots & 1 & \ddots & \ddots & \vdots & \vdots & \ddots & \ddots & \vdots \\
\vdots & \ddots & \ddots & \ddots & \ddots & \ddots & \ddots & \vdots & \vdots & \ddots & \ddots & 1 & \vdots & \ddots & \ddots & \vdots \\
\vdots & \ddots & \ddots & \ddots & \ddots & \ddots & \ddots & \vdots & 1 & \cdots & 1 & n+2 & 0 & \cdots & \cdots & 0 \\
\vdots & \ddots & \ddots & \ddots & \ddots & \ddots & \ddots & \vdots & 0 & \ddots & \ddots & 0 & m+2 & 1 & \cdots & 1 \\
\vdots & \ddots & \ddots & \ddots & \ddots & \ddots & \ddots & \vdots & \vdots & \ddots & \ddots & \vdots & 1 & \ddots & \ddots & \vdots \\
\vdots & \ddots & \ddots & \ddots & \ddots & \ddots & \ddots & 1 & \vdots & \ddots & \ddots & \vdots & \vdots & \ddots & \ddots & 1 \\
1 & \cdots & \cdots & \cdots & \cdots & \cdots & 1 & 0 & 0 & \cdots & \cdots & 0 & 1 & \cdots & 1 & m+2
\end{array}\right) \\
& =\frac{1}{4(m+n)^{2}} \operatorname{det}\left(\begin{array}{cccccccc}
n+2 & 2 & \cdots & 2 & 1 & \cdots & \cdots & 1 \\
2 & \ddots & \ddots & \vdots & \vdots & \ddots & \ddots & \vdots \\
\vdots & \ddots & \ddots & 2 & \vdots & \ddots & \ddots & \vdots \\
2 & \cdots & 2 & n+2 & 1 & \cdots & \cdots & 1 \\
1 & \cdots & \cdots & 1 & m+2 & 2 & \cdots & 2 \\
\vdots & \ddots & \ddots & \vdots & 2 & \ddots & \ddots & \vdots \\
\vdots & \ddots & \ddots & \vdots & \vdots & \ddots & \ddots & 2 \\
1 & \cdots & \cdots & 1 & 2 & \cdots & 2 & m+2
\end{array}\right) \\
& \times \operatorname{det}\left(\begin{array}{cccccccc}
n+2 & 0 & \cdots & 0 & -1 & \cdots & \cdots & -1 \\
0 & \ddots & \ddots & \vdots & \vdots & \ddots & \ddots & \vdots \\
\vdots & \ddots & \ddots & 0 & \vdots & \ddots & \ddots & \vdots \\
0 & \cdots & 0 & n+2 & -1 & \cdots & \cdots & -1 \\
-1 & \cdots & \cdots & -1 & m+2 & 0 & \cdots & 0 \\
\vdots & \ddots & \ddots & \vdots & 0 & \ddots & \ddots & \vdots \\
\vdots & \ddots & \ddots & \vdots & \vdots & \ddots & \ddots & 0 \\
-1 & \cdots & \cdots & -1 & 0 & \cdots & 0 & m+2
\end{array}\right) \\
& =\frac{1}{4(m+n)^{2}} \operatorname{det}\left(\begin{array}{cc}
A & B \\
B^{T} & C
\end{array}\right) \times \operatorname{det}\left(\begin{array}{cc}
D & E \\
E^{T} & F
\end{array}\right) \\
& =\frac{1}{4(m+n)^{2}} \times \operatorname{det} A \operatorname{det}\left(C-B^{T} A^{-1} B\right) \times \operatorname{det} D \operatorname{det}\left(F-E^{T} D^{-1} E\right) \\
& =\frac{1}{4(m+n)^{2}} \operatorname{det}\left(\begin{array}{cccc}
n+2 & 2 & \cdots & 2 \\
2 & \ddots & \ddots & \vdots \\
\vdots & \ddots & \ddots & 2 \\
2 & \cdots & 2 & n+2
\end{array}\right)_{m \times m}
\end{aligned}
$$




$$
\begin{aligned}
& \times \operatorname{det}\left(\begin{array}{cccc}
\frac{n(m+2)+m(2 m+3)}{n+2 m} & \frac{2 n+3 m}{n+2 m} & \cdots & \frac{2 n+3 m}{n+2 m} \\
\frac{2 n+3 m}{n+2 m} & \ddots & \ddots & \vdots \\
\vdots & \ddots & \ddots & \frac{2 n+3 m}{n+2 m} \\
\frac{2 n+3 m}{n+2 m} & \cdots & \frac{2 n+3 m}{n+2 m} & \frac{n(m+2)+m(2 m+3)}{n+2 m}
\end{array}\right)_{n \times n} \\
& \times \operatorname{det}\left(\begin{array}{cccc}
n+2 & 0 & \cdots & 0 \\
0 & \ddots & \ddots & \vdots \\
\vdots & \ddots & \ddots & 0 \\
0 & \cdots & 0 & n+2
\end{array}\right)_{m \times m} \\
& \times \operatorname{det}\left(\begin{array}{cccc}
\frac{n(m+2)+(m+4)}{n+2} & \frac{-m}{n+2} & \cdots & \frac{-m}{n+2} \\
\frac{-m}{n+2} & \ddots & \ddots & \vdots \\
\vdots & \ddots & \ddots & \frac{-m}{n+2} \\
\frac{-m}{n+2} & \cdots & \frac{-m}{n+2} & \frac{n(m+2)+(m+4)}{n+2}
\end{array}\right)_{n \times n} \\
& =\frac{1}{4(m+n)^{2}} \times 2^{m} \operatorname{det}\left(\begin{array}{cccc}
\frac{n+2}{2} & 1 & \cdots & 1 \\
1 & \ddots & \ddots & \vdots \\
\vdots & \ddots & \ddots & 1 \\
1 & \cdots & 1 & \frac{n+2}{2}
\end{array}\right)_{m \times m} \\
& \times\left(\frac{2 n+3 m}{n+2 m}\right)^{n} \operatorname{det}\left(\begin{array}{cccc}
\frac{n(m+2)+m(2 m+3)}{2 n+3 m} & 1 & \cdots & 1 \\
1 & \ddots & \ddots & \vdots \\
\vdots & \ddots & \ddots & 1 \\
1 & \cdots & 1 & \frac{n(m+2)+m(2 m+3)}{2 n+3 m}
\end{array}\right)_{n \times n} \\
& \times \operatorname{det}\left(\begin{array}{cccc}
n+2 & 0 & \cdots & 0 \\
0 & \ddots & \ddots & \vdots \\
\vdots & \ddots & \ddots & 0 \\
0 & \cdots & 0 & n+2
\end{array}\right)_{m \times m} \\
& \times\left(\frac{-m}{n+2}\right)^{n} \operatorname{det}\left(\begin{array}{cccc}
\frac{n(m+2)+(m+4)}{-m} & 1 & \cdots & 1 \\
1 & \ddots & \ddots & \vdots \\
\vdots & \ddots & \ddots & 1 \\
1 & \cdots & 1 & \frac{n(m+2)+(m+4)}{-m}
\end{array}\right)_{n \times n} \\
& =\frac{1}{4(m+n)^{2}} \times 2^{m} \times\left(\frac{n+2}{2}+m-1\right)\left(\frac{n+2}{2}-1\right)^{m-1} \\
& \times\left(\frac{2 n+3 m}{n+2 m}\right)^{n} \times\left(\frac{n(m+2)+m(2 m+3)}{2 n+3 m}+n-1\right)
\end{aligned}
$$




$$
\begin{aligned}
& \times\left(\frac{n(m+2)+m(2 m+3)}{2 n+3 m}-1\right)^{n-1} \times(n+2)^{m} \times\left(-\frac{m}{n+2}\right)^{n} \\
& \times\left(-\frac{n(m+2)+(m+4)}{m}+n-1\right) \times\left(-\frac{n(m+2)+(m+4)}{m}-1\right)^{n-1} .
\end{aligned}
$$

Thus,

$$
\begin{aligned}
\tau\left(K_{2} \times K_{m, n}\right)= & m^{n-1} n^{m-1}(m+2)^{m-1}(n+2)^{m-1} \\
& \times(n+m+2) .
\end{aligned}
$$

In particular,

$$
\tau\left(K_{2} \times K_{n, n}\right)=2 n^{2 n-2}(n+1)(n+2)^{2 n-2} ; \quad n \geq 1 .
$$

Theorem 6. For $m, n \geq 1$, we have

$$
\begin{aligned}
\tau\left(K_{3} \times K_{m, n}\right)= & 3 n^{m-1} m^{n-1}(m+3)^{2 n-2}(n+3)^{2 m-2} \\
& \times(n+m+3)^{2} .
\end{aligned}
$$

$$
\begin{aligned}
& \tau\left(K_{3} \times K_{m, n}\right) \\
& =\frac{1}{9(m+n)^{2}} \operatorname{det}(3(m+n) I-\bar{D}+\bar{A}) \\
& =\frac{1}{9(m+n)^{2}} \\
&
\end{aligned}
$$




$$
\begin{aligned}
& \begin{array}{cccccccccccc}
1 & \cdots & \cdots & 1 & 0 & 1 & \cdots & 1 & 1 & \cdots & \cdots & 1 \\
\vdots & \ddots & \ddots & \vdots & 1 & \ddots & \ddots & \vdots & \vdots & \ddots & \ddots & \vdots \\
\vdots & \ddots & \ddots & \vdots & \vdots & \ddots & \ddots & \vdots & \vdots & \ddots & \ddots & \vdots \\
1 & \cdots & \cdots & 1 & 1 & \cdots & 1 & 0 & 1 & \cdots & \cdots & 1 \\
0 & 1 & \cdots & 1 & 1 & \ldots & \cdots & 1 & 0 & 1 & \cdots & 1
\end{array}
\end{aligned}
$$

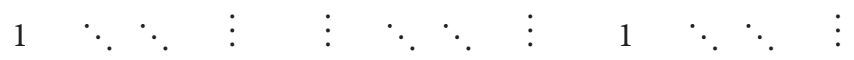

$$
\begin{aligned}
& \begin{array}{cccccccccccc}
\vdots & \ddots & \ddots & 1 & \vdots & \ddots & \ddots & \vdots & \vdots & \ddots & \ddots & 1 \\
1 & \cdots & 1 & 0 & 1 & \cdots & \cdots & 1 & 1 & \cdots & 1 & 0 \\
0 & \cdots & \cdots & 0 & 0 & 1 & \cdots & 1 & 1 & \ldots & \cdots & 1
\end{array}
\end{aligned}
$$

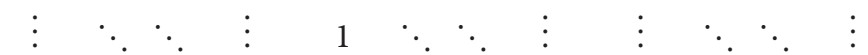

$$
\begin{aligned}
& \begin{array}{cccccccccccc}
\vdots & \ddots & \ddots & \vdots & \vdots & \ddots & \ddots & 1 & \vdots & \ddots & \ddots & \vdots \\
0 & \cdots & \cdots & 0 & 1 & \cdots & 1 & 0 & 1 & \cdots & \cdots & 1
\end{array} \\
& \begin{array}{llllllllllll}
m+3 & 1 & \cdots & 1 & 1 & \cdots & \cdots & 1 & 0 & 1 & \cdots & 1
\end{array}
\end{aligned}
$$

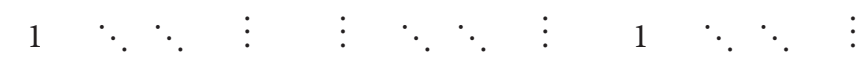

$$
\begin{aligned}
& \begin{array}{cccccccccccc}
\vdots & \ddots & \ddots & 1 & \vdots & \ddots & \ddots & \vdots & \vdots & \ddots & \ddots & 1 \\
1 & \cdots & 1 & m+3 & 1 & \ldots & \cdots & 1 & 1 & \cdots & 1 & 0
\end{array}
\end{aligned}
$$

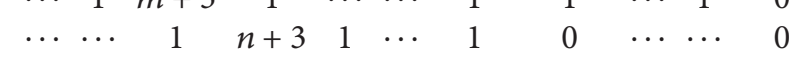

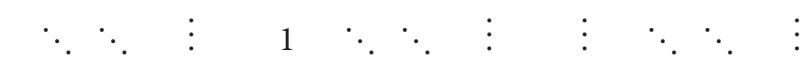

$$
\begin{aligned}
& \ddots \begin{array}{llllllllll}
\ddots & \vdots & \vdots & \ddots & \ddots & 1 & \vdots & \ddots & \ddots & \vdots
\end{array} \\
& \begin{array}{lllllllllll}
\cdots & \cdots & 1 & 1 & \cdots & 1 & n+3 & 0 & \cdots & \cdots & 0
\end{array} \\
& \begin{array}{llllllllllll}
0 & 1 & \cdots & 1 & 0 & \cdots & \cdots & 0 & m+3 & 1 & \cdots & 1
\end{array} \\
& \left.\begin{array}{cccccccccccc}
1 & \ddots & \ddots & \vdots & \vdots & \ddots & \ddots & \vdots & 1 & \ddots & \ddots & \vdots \\
\vdots & \ddots & \ddots & 1 & \vdots & \ddots & \ddots & \vdots & \vdots & \ddots & \ddots & 1 \\
1 & \cdots & 1 & 0 & 0 & \cdots & \cdots & 0 & 1 & \cdots & 1 & m+3
\end{array}\right) \\
& =\frac{1}{9(m+n)^{2}} \operatorname{det}\left(\begin{array}{ccc}
A & B & B \\
B & A & B \\
B & B & A
\end{array}\right)=\frac{1}{9(m+n)^{2}}[\operatorname{det}(A-B)]^{2}[\operatorname{det}(A+2 B)] \\
& =\frac{1}{9(m+n)^{2}}\left(\operatorname{det}\left(\begin{array}{cccccccc}
n+3 & 0 & \cdots & 0 & -1 & \cdots & \cdots & -1 \\
0 & \ddots & \ddots & \vdots & \vdots & \ddots & \ddots & \vdots \\
\vdots & \ddots & \ddots & 0 & \vdots & \ddots & \ddots & \vdots \\
0 & \cdots & 0 & n+3 & -1 & \cdots & \cdots & -1 \\
-1 & \cdots & \cdots & -1 & m+3 & 0 & \cdots & 0 \\
\vdots & \ddots & \ddots & \vdots & 0 & \ddots & \ddots & \vdots \\
\vdots & \ddots & \ddots & \vdots & \vdots & \ddots & \ddots & 0 \\
-1 & \cdots & \cdots & -1 & 0 & \cdots & 0 & m+3
\end{array}\right)\right)^{2} \\
& \times \operatorname{det}\left(\begin{array}{cccccccc}
n+3 & 3 & \cdots & 3 & 2 & \cdots & \cdots & 2 \\
3 & \ddots & \ddots & \vdots & \vdots & \ddots & \ddots & \vdots \\
\vdots & \ddots & \ddots & 3 & \vdots & \ddots & \ddots & \vdots \\
3 & \cdots & 3 & n+3 & 2 & \cdots & \cdots & 2 \\
2 & \cdots & \cdots & 2 & m+3 & 3 & \cdots & 3 \\
\vdots & \ddots & \ddots & \vdots & 3 & \ddots & \ddots & \vdots \\
\vdots & \ddots & \ddots & \vdots & \vdots & \ddots & \ddots & 3 \\
2 & \cdots & \cdots & 2 & 3 & \cdots & 3 & m+3
\end{array}\right)
\end{aligned}
$$




$$
\begin{aligned}
& =\frac{1}{9(m+n)^{2}}\left(\operatorname{det}\left(\begin{array}{cc}
A & B \\
B^{T} & C
\end{array}\right)\right)^{2} \times \operatorname{det}\left(\begin{array}{cc}
D & E \\
E^{T} & F
\end{array}\right) \\
& =\frac{1}{9(m+n)^{2}} \times(\operatorname{det} A)^{2}\left(\operatorname{det}\left(C-B^{T} A^{-1} B\right)\right)^{2} \times \operatorname{det} D \operatorname{det}\left(F-E^{T} D^{-1} E\right) .
\end{aligned}
$$

Thus,

$$
\begin{aligned}
& \tau\left(K_{3} \times K_{m, n}\right)=\frac{1}{9(m+n)^{2}}\left(\operatorname{det}\left(\begin{array}{cccc}
n+3 & 0 & \cdots & 0 \\
0 & \ddots & \ddots & \vdots \\
\vdots & \ddots & \ddots & 0 \\
0 & \cdots & 0 & n+3
\end{array}\right)_{m \times m}\right)^{2} \\
& \times\left(\operatorname{det}\left(\begin{array}{cccc}
\frac{n m+3 n+2 m+9}{n+3} & \frac{-m}{n+3} & \cdots & \frac{-m}{n+3} \\
\frac{-m}{n+3} & \ddots & \ddots & \vdots \\
\vdots & \ddots & \ddots & \frac{-m}{n+3} \\
\frac{-m}{n+3} & \cdots & \frac{-m}{n+3} & \frac{n m+3 n+2 m+9}{n+3}
\end{array}\right)_{n \times n}\right)^{2} \\
& \times \operatorname{det}\left(\begin{array}{cccc}
n+3 & 3 & \cdots & 3 \\
3 & \ddots & \ddots & \vdots \\
\vdots & \ddots & \ddots & 3 \\
3 & \cdots & 3 & n+3
\end{array}\right)_{m \times m} \\
& \times \operatorname{det}\left(\begin{array}{cccc}
\frac{n m+3 n+3 m^{2}+5 m}{n+3 m} & \frac{3 n+5 m}{n+3 m} & \ldots & \frac{3 n+5 m}{n+3 m} \\
\frac{3 n+5 m}{n+3 m} & \ddots & \ddots & \vdots \\
\vdots & \ddots & \ddots & \frac{3 n+5 m}{n+3 m} \\
\frac{3 n+5 m}{n+3 m} & \cdots & \frac{3 n+5 m}{n+3 m} & \frac{n m+3 n+3 m^{2}+5 m}{n+3 m}
\end{array}\right)_{n \times n} \\
& =\frac{1}{9(m+n)^{2}}(m+3)^{2 m} \times\left(\frac{-m}{n+3}\right)^{2 n} \\
& \times\left(\operatorname{det}\left(\begin{array}{cccc}
\frac{m n+3 n+2 m+9}{-m} & 1 & \ldots & 1 \\
1 & \ddots & \ddots & \vdots \\
\vdots & \ddots & \ddots & 1 \\
1 & \ldots & 1 & \frac{m n+3 n+2 m+9}{-m}
\end{array}\right)\right)^{2} \\
& \times 3^{m} \times \operatorname{det}\left(\begin{array}{cccc}
\frac{n+3}{3} & 1 & \ldots & 1 \\
1 & \ddots & \ddots & \vdots \\
\vdots & \ddots & \ddots & 1 \\
1 & \cdots & 1 & \frac{n+3}{3}
\end{array}\right)_{m \times m} \times\left(\frac{3 n+5 m}{n+3 m}\right)^{n}
\end{aligned}
$$




$$
\times \operatorname{det}\left(\begin{array}{cccc}
\frac{m n+3 n+2 m^{2}+5 m}{3 n+5 m} & 1 & \ldots & 1 \\
1 & \ddots & \ddots & \vdots \\
\vdots & \ddots & \ddots & 1 \\
1 & \ldots & 1 & \frac{m n+3 n+2 m^{2}+5 m}{3 n+5 m}
\end{array}\right) .
$$

Using Lemma 2, we have

$$
\begin{aligned}
\tau & \left(K_{3} \times K_{m, n}\right) \\
= & \frac{1}{9(m+n)^{2}} \times(n+3)^{2 m} \times\left(\frac{-m}{n+3}\right)^{2 n} \\
& \times\left[-\frac{n m+3 n+2 m+9}{m}+n-1\right]^{2} \\
& \times\left[-\frac{n m+3 n+2 m+9}{m}-1\right]^{2 n-2} \times 3^{m}\left(\frac{n+3}{3}+m-1\right) \\
\times & \left(\frac{n+3}{3}-1\right)^{m-1} \times\left(\frac{3 n+5 m}{n+3 m}\right)^{n} \\
& \times\left[\frac{n m+3 n+3 m^{2}+5 m}{3 n+5 m}+n-1\right] \\
& \times\left[\frac{n m+3 n+3 m^{2}+5 m}{3 n+5 m}-1\right]^{n-1} \\
= & \frac{1}{9(m+n)^{2}}(n+3)^{2 m} \\
& \times\left[\frac{1}{(n+3)^{2 n}} \times(3 n+3 m+9)^{2}\right. \\
& \left.\times(n m+3 n+3 m+9)^{2 n-2}\right]
\end{aligned}
$$

$$
\begin{aligned}
\times\left[(n+3 m) \times n^{m-1} \times \frac{1}{(n+3 m)^{n}}\right. \\
\times\left(6 n m+3 n^{2}+3 m^{2}\right) \\
\left.\times\left(n m+3 m^{2}\right)^{n-1}\right] \\
=3 n^{m-1} m^{n-1}(m+3)^{2 n-2}(n+3)^{2 m-2}(n+m+3)^{2} .
\end{aligned}
$$

In particular,

$$
\tau\left(K_{3} \times K_{n, n}\right)=3 n^{2 n-2}(2 n+3)^{2}(n+3)^{4 n-4} ; \quad n \geq 1 .
$$

\section{Number of Spanning Trees of Normal Product of Graphs}

The normal product, or the strong product, $G_{1} \circ G_{2}$, is the simple graph with $V\left(G_{1} \circ G_{2}\right)=V_{1} \times V_{2}$, where $\left(u_{1}, u_{2}\right)$ and $\left(v_{1}, v_{2}\right)$ are adjacent in $G_{1} \circ G_{2}$ if and only if either $u_{1}=v_{1}$ and $u_{2}$ is adjacent to $v_{2}, u_{1}$ is adjacent to $v_{1}$ and $u_{2}=v_{2}$, or $u_{1}$ is adjacent to $v_{1}$ and $u_{2}$ is adjacent to $v_{2}$ [13].

Theorem 7. For $n, m \geq 1$, we have

$$
\begin{aligned}
\tau\left(K_{2} \circ K_{m, n}\right)= & 2^{2 m+2 n-2} \times n^{m-1} \\
& \times m^{n-1} \times(n+1)^{m} \times(m+1)^{n} .
\end{aligned}
$$

Proof. Applying Lemma 1, we have

$$
\begin{aligned}
& \tau\left(K_{2} \circ K_{m, n}\right) \\
& =\frac{1}{4(m+n)^{2}} \operatorname{det}(2(m+n) I-\bar{D}+\bar{A}) \\
& =\frac{1}{4(m+n)^{2}}
\end{aligned}
$$


$\times \operatorname{det}\left(\begin{array}{cccccccccccccccc}2 n+2 & 1 & \cdots & 1 & 0 & \cdots & \cdots & 0 & 0 & 1 & \cdots & 1 & 0 & \cdots & \cdots & 0 \\ 1 & \ddots & \ddots & \vdots & \vdots & \ddots & \ddots & \vdots & 1 & \ddots & \ddots & \vdots & \vdots & \ddots & \ddots & \vdots \\ \vdots & \ddots & \ddots & 1 & \vdots & \ddots & \ddots & \vdots & \vdots & \ddots & \ddots & 1 & \vdots & \ddots & \ddots & \vdots \\ 1 & \cdots & 1 & 2 n+2 & 0 & \cdots & \cdots & 0 & 1 & \cdots & 1 & 0 & 0 & \cdots & \cdots & 0 \\ 0 & \cdots & \cdots & 0 & 2 m+2 & 1 & \cdots & 1 & 0 & \cdots & \cdots & 0 & 0 & 1 & \cdots & 1 \\ \vdots & \ddots & \ddots & 1 & 1 & \ddots & \ddots & \vdots & \vdots & \ddots & \ddots & \vdots & 1 & \ddots & \ddots & \vdots \\ \vdots & \ddots & \ddots & \vdots & \vdots & \ddots & \ddots & 1 & \vdots & \ddots & \ddots & \vdots & \vdots & \ddots & \ddots & 1 \\ 0 & \cdots & \cdots & 0 & 1 & \cdots & 1 & 2 m+2 & 0 & \cdots & \cdots & 0 & 1 & \cdots & 1 & 0 \\ 0 & 1 & \cdots & 1 & 0 & \cdots & \cdots & 0 & 2 n+2 & 1 & \cdots & 1 & 0 & \cdots & \cdots & 0 \\ 1 & \ddots & \ddots & \vdots & \vdots & \ddots & \ddots & \vdots & 1 & \ddots & \ddots & \vdots & \vdots & \ddots & \ddots & \vdots \\ \vdots & \ddots & \ddots & 1 & \vdots & \ddots & \ddots & \vdots & \vdots & \ddots & \ddots & 1 & \vdots & \ddots & \ddots & \vdots \\ 1 & \cdots & 1 & 0 & 0 & \cdots & \cdots & 0 & 1 & \cdots & 1 & 2 n+2 & 0 & \cdots & \cdots & 0 \\ 0 & \cdots & \cdots & 0 & 0 & 1 & \cdots & 1 & 0 & \cdots & \cdots & 0 & 2 m+2 & 1 & \cdots & 1 \\ \vdots & \ddots & \ddots & \vdots & 1 & \ddots & \ddots & \vdots & \vdots & \ddots & \ddots & \vdots & 1 & \ddots & \ddots & \vdots \\ \vdots & \ddots & \ddots & \vdots & \vdots & \ddots & \ddots & 1 & \vdots & \ddots & \ddots & \vdots & \vdots & \ddots & \ddots & 1 \\ 0 & \cdots & \cdots & 0 & 1 & \cdots & 1 & 0 & 0 & \cdots & \cdots & 0 & 1 & \cdots & 1 & 2 m+2\end{array}\right)$

$=\frac{1}{4(m+n)^{2}} \operatorname{det}\left(\begin{array}{cccccccc}2 n+2 & 2 & \cdots & 2 & 0 & \cdots & \cdots & 0 \\ 2 & \ddots & \ddots & \vdots & \vdots & \ddots & \ddots & \vdots \\ \vdots & \ddots & \ddots & 2 & \vdots & \ddots & \ddots & \vdots \\ 2 & \cdots & 2 & 2 n+2 & 0 & \cdots & \cdots & 0 \\ 0 & \cdots & \cdots & 0 & 2 m+2 & 2 & \cdots & 2 \\ \vdots & \ddots & \ddots & \vdots & 2 & \ddots & \ddots & \vdots \\ \vdots & \ddots & \ddots & \vdots & \vdots & \ddots & \ddots & 2 \\ 0 & \cdots & \cdots & 0 & 2 & \cdots & 2 & 2 m+2\end{array}\right)$

$\times \operatorname{det}\left(\begin{array}{cccccccc}2 n+2 & 0 & \cdots & 0 & 0 & \cdots & \cdots & 0 \\ 0 & \ddots & \ddots & \vdots & \vdots & \ddots & \ddots & \vdots \\ \vdots & \ddots & \ddots & 0 & \vdots & \ddots & \ddots & \vdots \\ 0 & \cdots & 0 & 2 n+2 & 0 & \cdots & \cdots & 0 \\ 0 & \cdots & \cdots & 0 & 2 m+2 & 0 & \cdots & 0 \\ \vdots & \ddots & \ddots & \vdots & 0 & \ddots & \ddots & \vdots \\ \vdots & \ddots & \ddots & \vdots & \vdots & \ddots & \ddots & 0 \\ 0 & \cdots & \cdots & 0 & 0 & \cdots & 0 & 2 m+2\end{array}\right)$

$=\frac{1}{4(m+n)^{2}} \operatorname{det}\left(\begin{array}{cccc}2 n+2 & 2 & \cdots & 2 \\ 2 & \ddots & \ddots & \vdots \\ \vdots & \ddots & \ddots & 2 \\ 2 & \cdots & 2 & 2 n+2\end{array}\right)_{m \times m} \times \operatorname{det}\left(\begin{array}{cccc}2 m+2 & 2 & \cdots & 2 \\ 2 & \ddots & \ddots & \vdots \\ \vdots & \ddots & \ddots & 2 \\ 2 & \cdots & 2 & 2 m+2\end{array}\right)_{n \times n}$

$\times \operatorname{det}\left(\begin{array}{cccc}2 n+2 & 0 & \cdots & 0 \\ 0 & \ddots & \ddots & \vdots \\ \vdots & \ddots & \ddots & 0 \\ 0 & \cdots & 0 & 2 n+2\end{array}\right)_{m \times m} \times \operatorname{det}\left(\begin{array}{cccc}2 m+2 & 0 & \cdots & 0 \\ 0 & \ddots & \ddots & \vdots \\ \vdots & \ddots & \ddots & 0 \\ 0 & \cdots & 0 & 2 m+2\end{array}\right)_{n \times n}$ 


$$
\begin{aligned}
& =\frac{1}{4(m+n)^{2}} \times 2^{m}(n+m) n^{m-1} \times 2^{n}(n+m) m^{n-1} \times 2^{m}(n+1)^{m} \times 2^{n}(m+1)^{n} \\
& =2^{2 m+2 n-2} \times n^{m-1} \times m^{n-1} \times(n+1)^{m} \times(m+1)^{n} .
\end{aligned}
$$

In particular,

$$
\tau\left(K_{2} \circ K_{n, n}\right)=2^{4 n-2} \times n^{2 n-2} \times(n+1)^{2 n} ; \quad n \geq 1 .
$$

$$
\begin{aligned}
\tau\left(K_{3} \circ K_{m, n}\right)= & 3^{3 m+3 n-2} \times n^{m-1} \times m^{n-1} \\
& \times(n+1)^{2 m} \times(m+1)^{2 n}
\end{aligned}
$$

Theorem 8. For $m, n \geq 1$, we have

$$
\begin{aligned}
& \tau\left(K_{3} \circ K_{m, n}\right) \\
& =\frac{1}{9(m+n)^{2}} \operatorname{det}(3(m+n) I-\bar{D}+\bar{A}) \\
& =\frac{1}{9(m+n)^{2}} \\
& \left(\begin{array}{cccccccccccc}
3 n+3 & 1 & \cdots & 1 & 0 & \cdots & \cdots & 0 & 0 & 1 & \cdots & 1 \\
1 & \ddots & \ddots & \vdots & \vdots & \ddots & \ddots & \vdots & 1 & \ddots & \ddots & \vdots \\
\vdots & \ddots & \ddots & 1 & \vdots & \ddots & \ddots & \vdots & \vdots & \ddots & \ddots & 1 \\
1 & \cdots & 1 & 3 n+3 & 0 & \cdots & \cdots & 0 & 1 & \cdots & 1 & 0 \\
0 & \cdots & \cdots & 0 & 3 m+3 & 1 & \cdots & 1 & 0 & \cdots & \cdots & 0 \\
\vdots & \ddots & \ddots & \vdots & 1 & \ddots & \ddots & \vdots & \vdots & \ddots & \ddots & \vdots \\
\vdots & \ddots & \ddots & \vdots & \vdots & \ddots & \ddots & \vdots & \vdots & \ddots & \ddots & \vdots \\
0 & \cdots & \cdots & 0 & 1 & \cdots & 1 & 3 m+3 & 0 & \cdots & \cdots & 0 \\
0 & 1 & \cdots & 1 & 0 & \cdots & \cdots & 0 & 3 n+3 & 1 & \cdots & 1 \\
1 & \ddots & \ddots & \vdots & \vdots & \ddots & \ddots & \vdots & 1 & \ddots & \ddots & \vdots \\
\vdots & \ddots & \ddots & 1 & \vdots & \ddots & \ddots & \vdots & \vdots & \ddots & \ddots & 1 \\
1 & \cdots & 1 & 0 & 0 & \cdots & \cdots & 0 & 1 & \cdots & 1 & 3 n+3 \\
0 & \cdots & \cdots & 0 & 0 & 1 & \cdots & 1 & 0 & \cdots & \cdots & 0 \\
\vdots & \ddots & \ddots & \vdots & 1 & \ddots & \ddots & \vdots & \vdots & \ddots & \ddots & \vdots \\
\vdots & \ddots & \ddots & \vdots & \vdots & \ddots & \ddots & 1 & \vdots & \ddots & \ddots & \vdots \\
0 & \cdots & \cdots & 0 & 1 & \cdots & 1 & 0 & 0 & \cdots & \cdots & 0 \\
0 & 1 & \cdots & 1 & 0 & \cdots & \cdots & 0 & 0 & 1 & \cdots & 1 \\
1 & \ddots & \ddots & \vdots & \vdots & \ddots & \ddots & \vdots & 1 & \ddots & \ddots & \vdots \\
\vdots & \ddots & \ddots & 1 & \vdots & \ddots & \ddots & \vdots & \vdots & \ddots & \ddots & 1 \\
1 & \cdots & 1 & 0 & 0 & \cdots & \cdots & 0 & 1 & \cdots & 1 & 0 \\
0 & \cdots & \cdots & 0 & 0 & 1 & \cdots & 1 & 0 & \cdots & \cdots & 0 \\
\vdots & \ddots & \ddots & \vdots & 1 & \ddots & \ddots & \vdots & \vdots & \ddots & \ddots & \vdots \\
\vdots & \ddots & \ddots & \vdots & \vdots & \ddots & \ddots & 1 & \vdots & \ddots & \ddots & \vdots \\
0 & \cdots & \cdots & 0 & 1 & \cdots & 1 & 0 & 0 & \cdots & \cdots & 0
\end{array}\right.
\end{aligned}
$$




$$
\begin{aligned}
& \left.\begin{array}{cccccccccccc}
\vdots & \ddots & \ddots & \vdots & 1 & \ddots & \ddots & \vdots & \vdots & \ddots & \ddots & \vdots \\
\vdots & \ddots & \ddots & \vdots & \vdots & \ddots & \ddots & \vdots & \vdots & \ddots & \ddots & \vdots \\
0 & \cdots & \cdots & 0 & 1 & \cdots & 1 & 0 & 0 & \cdots & \cdots & 0 \\
0 & 1 & \cdots & 1 & 0 & \cdots & \cdots & 0 & 0 & 1 & \cdots & 1 \\
1 & \ddots & \ddots & \vdots & \vdots & \ddots & \ddots & \vdots & 1 & \ddots & \ddots & \vdots \\
\vdots & \ddots & \ddots & 1 & \vdots & \ddots & \ddots & \vdots & \vdots & \ddots & \ddots & 1 \\
1 & \cdots & 1 & 0 & 0 & \cdots & \cdots & 0 & 1 & \cdots & 1 & 0 \\
0 & \cdots & \cdots & 0 & 0 & 1 & \cdots & 1 & 0 & \cdots & \cdots & 0 \\
\vdots & \ddots & \ddots & \vdots & 1 & \ddots & \ddots & \vdots & \vdots & \ddots & \ddots & \vdots \\
\vdots & \ddots & \ddots & \vdots & \vdots & \ddots & \ddots & 1 & \vdots & \ddots & \ddots & \vdots \\
0 & \cdots & \cdots & 0 & 1 & \cdots & 1 & 0 & 0 & \cdots & \cdots & 0 \\
m+3 & 1 & \cdots & 1 & 0 & \cdots & \cdots & 0 & 0 & 1 & \cdots & 1 \\
1 & \ddots & \ddots & \vdots & \vdots & \ddots & \ddots & \vdots & 1 & \ddots & \ddots & \vdots \\
\vdots & \ddots & \ddots & 1 & \vdots & \ddots & \ddots & \vdots & \vdots & \ddots & \ddots & 1 \\
1 & \cdots & 1 & 3 m+3 & 0 & \cdots & \cdots & 0 & 1 & \cdots & 1 & 0 \\
0 & \cdots & \cdots & 0 & 3 n+3 & 1 & \cdots & 1 & 0 & \cdots & \cdots & 0 \\
\vdots & \ddots & \ddots & \vdots & 1 & \ddots & \ddots & \vdots & \vdots & \ddots & \ddots & \vdots \\
\vdots & \ddots & \ddots & \vdots & \vdots & \ddots & \ddots & 1 & \vdots & \ddots & \ddots & \vdots \\
0 & \cdots & \cdots & 0 & 1 & \cdots & 1 & 3 n+3 & 0 & \cdots & \cdots & 0 \\
0 & 1 & \cdots & 1 & 0 & \cdots & \cdots & 0 & 3 m+3 & 1 & \cdots & 1 \\
1 & \ddots & \ddots & \vdots & \vdots & \ddots & \ddots & \vdots & 1 & \ddots & \ddots & \vdots \\
\vdots & \ddots & \ddots & 1 & \vdots & \ddots & \ddots & \vdots & \vdots & \ddots & \ddots & 1 \\
1 & \cdots & 1 & 0 & 0 & \cdots & \cdots & 0 & 1 & \cdots & 1 & 3 m+3
\end{array}\right) \\
& =\frac{1}{9(m+n)^{2}} \operatorname{det}\left(\begin{array}{ccc}
A & B & B \\
B & A & B \\
B & B & A
\end{array}\right)=\frac{1}{9(m+n)^{2}}[\operatorname{det}(A-B)]^{2}[\operatorname{det}(A+2 B)] \\
& =\frac{1}{9(m+n)^{2}}\left(\operatorname{det}\left(\begin{array}{cccccccc}
3 n+3 & 0 & \cdots & 0 & 0 & \cdots & \cdots & 0 \\
0 & \ddots & \ddots & \vdots & \vdots & \ddots & \ddots & \vdots \\
\vdots & \ddots & \ddots & 0 & \vdots & \ddots & \ddots & \vdots \\
0 & \cdots & 0 & 3 n+3 & 0 & \cdots & \cdots & 0 \\
0 & \cdots & \cdots & 0 & 3 m+3 & 0 & \cdots & 0 \\
\vdots & \ddots & \ddots & \vdots & 0 & \ddots & \ddots & \vdots \\
\vdots & \ddots & \ddots & \vdots & \vdots & \ddots & \ddots & 0 \\
0 & \cdots & \cdots & 0 & 0 & \cdots & 0 & 3 m+3
\end{array}\right)\right)^{2} \\
& \times \operatorname{det}\left(\begin{array}{cccccccc}
3 n+3 & 3 & \cdots & 3 & 0 & \cdots & \cdots & 0 \\
3 & \ddots & \ddots & \vdots & \vdots & \ddots & \ddots & \vdots \\
\vdots & \ddots & \ddots & 3 & \vdots & \ddots & \ddots & \vdots \\
3 & \cdots & 3 & 3 n+3 & 0 & \cdots & \cdots & 0 \\
0 & \cdots & \cdots & 0 & 3 m+3 & 3 & \cdots & 3 \\
\vdots & \ddots & \ddots & \vdots & 3 & \ddots & \ddots & \vdots \\
\vdots & \ddots & \ddots & \vdots & \vdots & \ddots & \ddots & 3 \\
0 & \cdots & \cdots & 0 & 3 & \cdots & 3 & 3 m+3
\end{array}\right)
\end{aligned}
$$




$$
\begin{aligned}
& =\frac{1}{9(m+n)^{2}}\left(\operatorname{det}\left(\begin{array}{cccc}
3 n+3 & 0 & \cdots & 0 \\
0 & \ddots & \ddots & \vdots \\
\vdots & \ddots & \ddots & 0 \\
0 & \cdots & 0 & 3 n+3
\end{array}\right)_{m \times m}\right)^{2} \\
& \times\left(\operatorname{det}\left(\begin{array}{cccc}
3 m+3 & 0 & \cdots & 0 \\
0 & \ddots & \ddots & \vdots \\
\vdots & \ddots & \ddots & 0 \\
0 & \cdots & 0 & 3 m+3
\end{array}\right)_{n \times n}\right)^{2} \\
& \times \operatorname{det}\left(\begin{array}{cccc}
3 n+3 & 3 & \cdots & 3 \\
3 & \ddots & \ddots & \vdots \\
\vdots & \ddots & \ddots & 3 \\
3 & \cdots & 3 & 3 n+3
\end{array}\right) \times \operatorname{det}\left(\begin{array}{cccc}
3 m+3 & 3 & \cdots & 3 \\
3 & \ddots & \ddots & \vdots \\
\vdots & \ddots & \ddots & 3 \\
3 & \cdots & 3 & 3 m+3
\end{array}\right) \text { }
\end{aligned}
$$

Using Lemma 2, we have

$$
\begin{aligned}
\tau\left(K_{3} \circ K_{m, n}\right)= & \frac{1}{9(m+n)^{2}} \times(3 n+3)^{2 m} \times(3 m+3)^{2 n} \\
& \times\left(3^{m} \times(n+m) \times n^{m-1}\right) \\
& \times\left(3^{n} \times(n+m) \times m^{n-1}\right) \\
= & 3^{3 m+3 n-2} \times n^{m-1} \times m^{n-1} \\
& \times(n+1)^{2 m} \times(m+1)^{2 n} .
\end{aligned}
$$

\section{Number of Spanning Trees of Composition Product of Graphs}

The composition, or lexicographic product, $G_{1}\left[G_{2}\right]$, is the simple graph with $V_{1} \times V_{2}$ as the vertex set in which the vertices $\left(u_{1}, u_{2}\right)$ and $\left(v_{1}, v_{2}\right)$ are adjacent if either $u_{1}$ is adjacent to $v_{1}$ or $u_{1}=v_{1}$ and $u_{2}$ is adjacent to $v_{2}$ in $G_{2}$ [13].

Theorem 9. For $n, m \geq 1$, we have

$$
\begin{aligned}
\tau\left(K_{2}\left[K_{m, n}\right]\right)= & 4(m+n)^{2} \\
& \times(m+2 n)^{2 m-2}(n+2 m)^{2 n-2} .
\end{aligned}
$$

In paricular,

$$
\tau\left(K_{3} \circ K_{n, n}\right)=3^{6 n-2} \times n^{2 n-2} \times(n+1)^{4 n} ; \quad n \geq 1 .
$$

Proof. Applying Lemma 1, we have

$$
\begin{aligned}
& \tau\left(K_{2}\left[K_{m, n}\right]\right) \\
& =\frac{1}{4(m+n)^{2}} \operatorname{det}(2(m+n) I-\bar{D}+\bar{A}) \\
& =\frac{1}{4(m+n)^{2}}
\end{aligned}
$$




$$
\begin{aligned}
& \times \operatorname{det}\left(\begin{array}{cccccccc}
m+2 n+1 & 1 & \cdots & 1 & 0 & \cdots & \cdots & 0 \\
1 & \ddots & \ddots & \vdots & \vdots & \ddots & \ddots & \vdots \\
\vdots & \ddots & \ddots & 1 & \vdots & \ddots & \ddots & \vdots \\
1 & \cdots & 1 & m+2 n+1 & 0 & \cdots & \cdots & 0 \\
0 & \cdots & \cdots & 0 & n+2 m+1 & 1 & \cdots & 1 \\
\vdots & \ddots & \ddots & \vdots & 1 & \ddots & \ddots & \vdots \\
\vdots & \ddots & \ddots & \vdots & \vdots & \ddots & \ddots & 1 \\
0 & \cdots & \cdots & 0 & 1 & \cdots & 1 & n+2 m+1 \\
0 & \cdots & \cdots & 0 & 0 & \cdots & \cdots & 0 \\
\vdots & \ddots & \ddots & \vdots & \vdots & \ddots & \ddots & \vdots \\
\vdots & \ddots & \ddots & \vdots & \vdots & \ddots & \ddots & \vdots \\
0 & \cdots & \cdots & 0 & 0 & \cdots & \cdots & 0 \\
0 & \cdots & \cdots & 0 & 0 & \cdots & \cdots & 0 \\
\vdots & \ddots & \ddots & \vdots & \vdots & \ddots & \ddots & \vdots \\
\vdots & \ddots & \ddots & \vdots & \vdots & \ddots & \ddots & \vdots \\
0 & \cdots & \cdots & 0 & 0 & \cdots & \cdots & 0
\end{array}\right.
\end{aligned}
$$

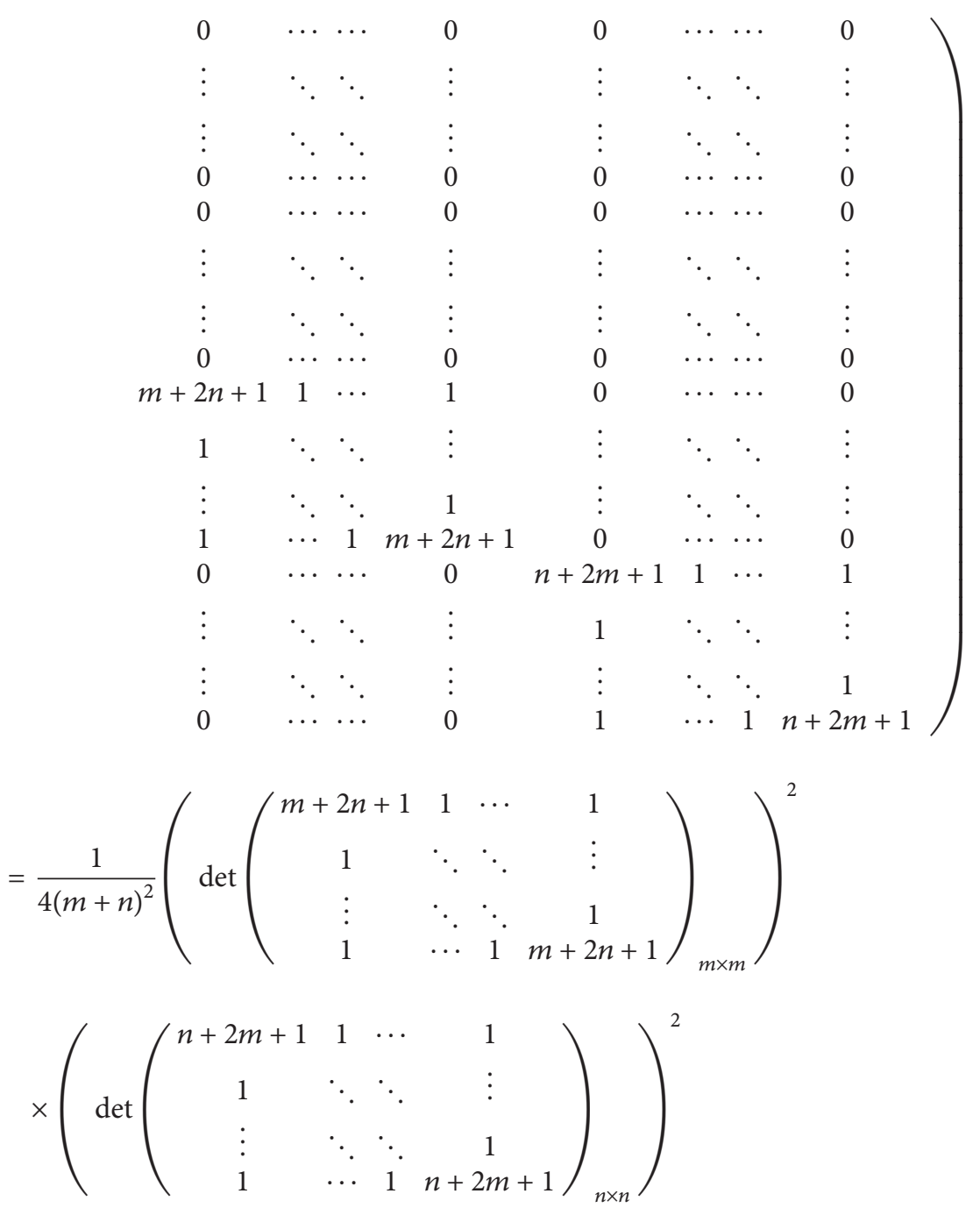




$$
\begin{aligned}
& =\frac{1}{4(m+n)^{2}}(2 n+2 m)^{2}(m+2 n)^{2 m-2} \times(2 n+2 m)^{2}(n+2 m)^{2 n-2} \\
& =4(m+n)^{2}(m+2 n)^{2 m-2}(n+2 m)^{2 n-2} .
\end{aligned}
$$

In particular,

$$
\tau\left(K_{2}\left[K_{n, n}\right]\right)=16 \times 3^{4 n-4} \times n^{4 n-4} ; \quad n \geq 1 .
$$

Theorem 10. For $m, n \geq 1$, we have

$$
\tau\left(K_{3}\left[K_{m, n}\right]\right)=3^{4}(m+n)^{4}(3 m+2 n)^{3 n-3}(3 n+2 m)^{3 m-3} .
$$

Proof. Applying Lemma 1, we have

$$
\begin{aligned}
& \tau\left(K_{3}\left[K_{m, n}\right]\right) \\
& =\frac{1}{9(m+n)^{2}} \operatorname{det}(3(m+n) I-\bar{D}+\bar{A}) \\
& =\frac{1}{9(m+n)^{2}}
\end{aligned}
$$

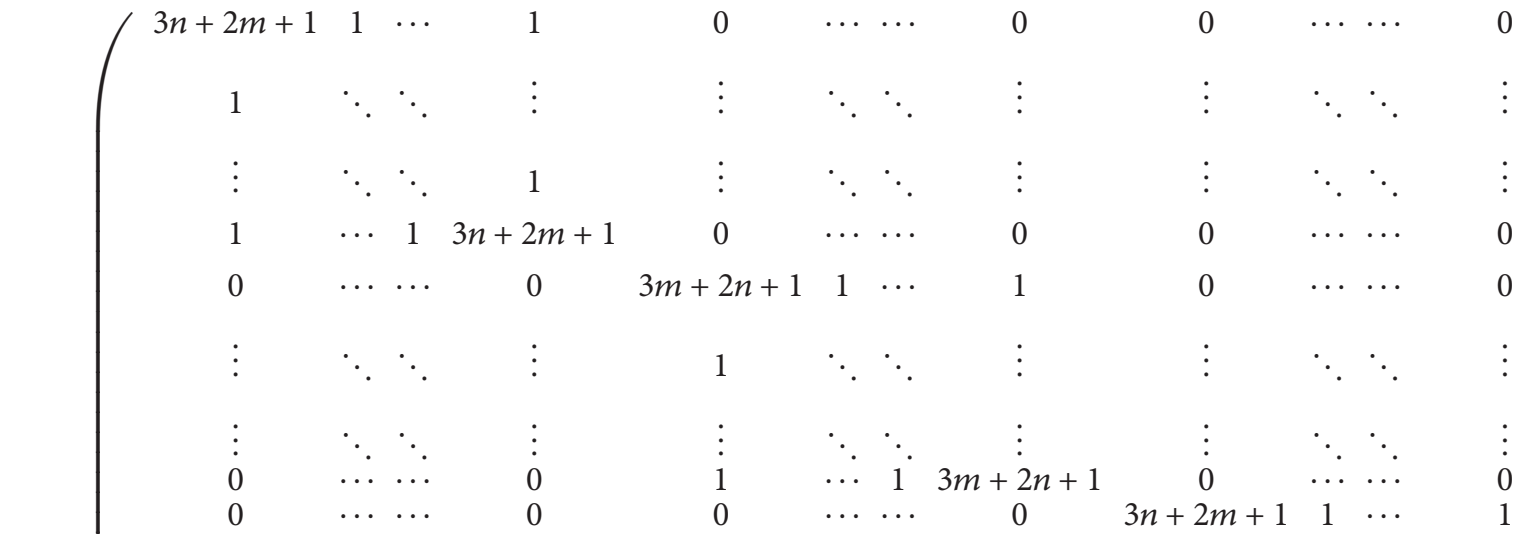

$$
\begin{aligned}
& \begin{array}{cccccccccccr}
\vdots & \ddots & \ddots & \vdots & \vdots & \ddots & \ddots & \vdots & 1 & \ddots & \ddots & \vdots \\
\vdots & \ddots & \ddots & \vdots & \vdots & \ddots & \ddots & \vdots & \vdots & \ddots & \ddots & 1
\end{array} \\
& \begin{array}{lll}
0 & \cdots & \cdots
\end{array} \\
& \begin{array}{llllllllll}
\vdots & \ddots & \ddots & \vdots & \ddots & \ddots & \vdots & \ddots & \ddots
\end{array} \\
& \begin{array}{cccccccccccc}
\vdots & \ddots & \ddots & \vdots & \vdots & \ddots & \ddots & \vdots & \vdots & \ddots & \ddots & \vdots \\
0 & \cdots & \cdots & 0 & 0 & \ldots & \cdots & 0 & 0 & \ldots & \cdots & 0 \\
0 & \cdots & \cdots & 0 & 0 & \ldots & \ldots & 0 & 0 & \ldots & \ldots & 0
\end{array} \\
& \begin{array}{llllllll}
\vdots & \ddots & \ddots & \vdots & \ddots & \ddots & \vdots & \ddots
\end{array} \\
& \begin{array}{llllllllllll}
\vdots & \ddots & \ddots & \vdots & \vdots & \ddots & \ddots & \vdots & \vdots & \ddots & \ddots & \vdots \\
0 & \cdots & \cdots & 0 & 0 & \cdots & \cdots & 0 & 0 & \cdots & \cdots & 0
\end{array} \\
& \begin{array}{llllllllll}
0 & \ldots & \ldots & 0 & 0 & \ldots & \ldots & 0 & 0 & \ldots
\end{array}
\end{aligned}
$$

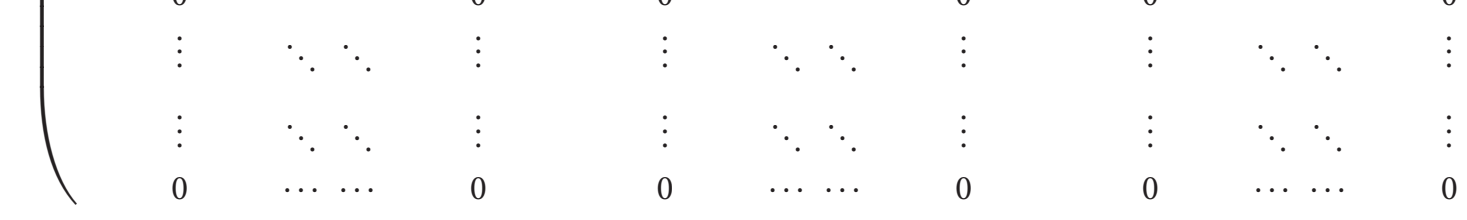




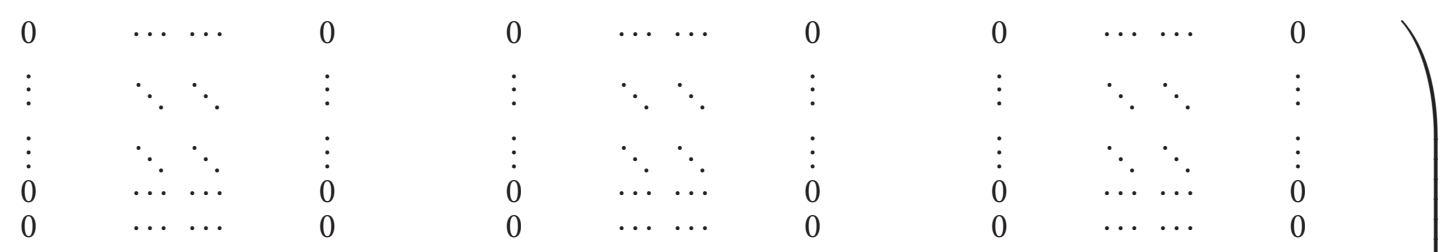

$$
\begin{aligned}
& \begin{array}{llllllllll}
\vdots & \ddots & \ddots & \vdots & \vdots & \ddots & \ddots & \vdots & \vdots & \ddots
\end{array}
\end{aligned}
$$

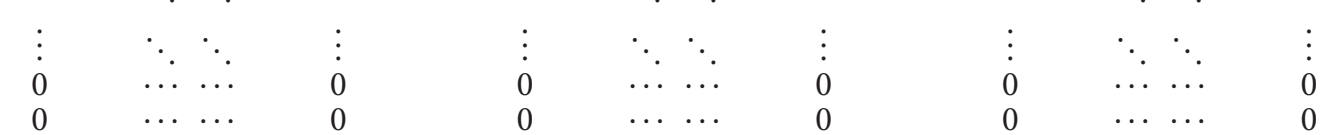

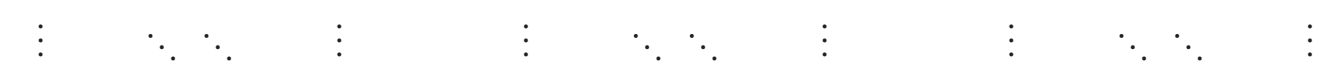

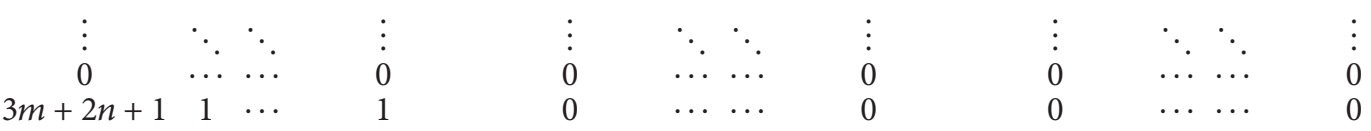

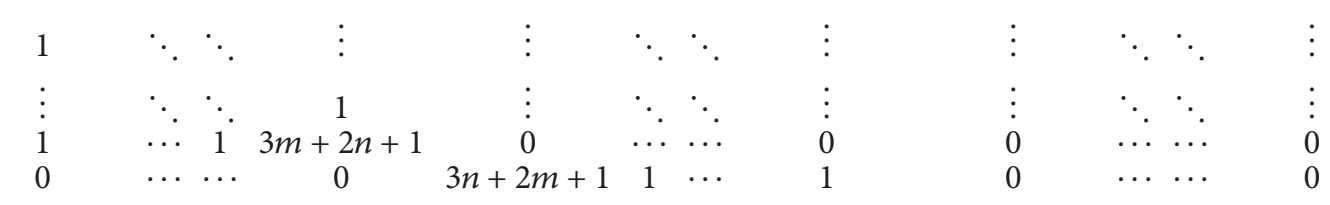

$$
\begin{aligned}
& \begin{array}{llllllllll}
\vdots & \ddots & \vdots & 1 & \ddots & \ddots & \vdots & \vdots & \ddots & \vdots
\end{array} \\
& \begin{array}{cccccccccccc}
\vdots & \ddots & \ddots & \vdots & \vdots & \ddots & \ddots & 1 & \vdots & \ddots & \ddots & \vdots \\
0 & \cdots & \cdots & 0 & 1 & \cdots & 1 & 3 n+2 m+1 & 0 & \cdots & \cdots & 0 \\
0 & \cdots & \cdots & 0 & 0 & \cdots & \cdots & 0 & 3 m+2 n+1 & 1 & \cdots &
\end{array}
\end{aligned}
$$

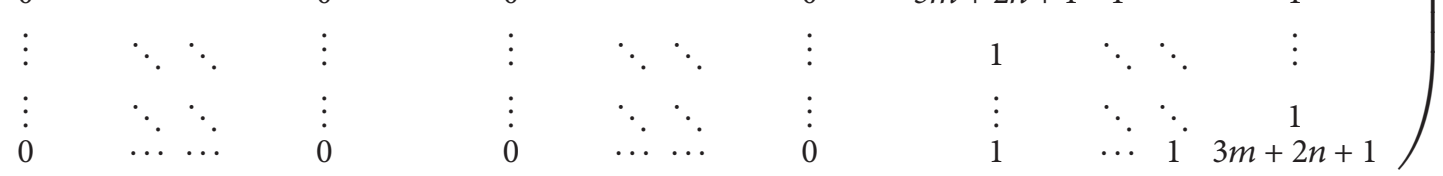

$$
\begin{aligned}
& \left.=\frac{1}{9(m+n)^{2}} \operatorname{det}\left(\begin{array}{cccccccc}
3 n+2 m+1 & 1 & \cdots & 1 & 0 & \cdots & \cdots & 0 \\
1 & \ddots & \ddots & \vdots & \vdots & \ddots & \ddots & \vdots \\
\vdots & \ddots & \ddots & 1 & \vdots & \ddots & \ddots & \vdots \\
1 & \cdots & 1 & 3 n+2 m+1 & 0 & \cdots & \cdots & 0 \\
0 & \cdots & \cdots & 0 & 3 m+2 n+1 & 1 & \cdots & 1 \\
\vdots & \ddots & \ddots & \vdots & 1 & \ddots & \ddots & \vdots \\
\vdots & \ddots & \ddots & \vdots & \vdots & \ddots & \ddots & 1 \\
0 & \cdots & \cdots & 0 & 1 & \cdots & 1 & 3 m+2 n+1
\end{array}\right)\right)^{3} \\
& =\frac{1}{9(m+n)^{2}}\left(\operatorname{det}\left(\begin{array}{cccc}
3 n+2 m+1 & 1 & \cdots & 1 \\
1 & \ddots & \ddots & \vdots \\
\vdots & \ddots & \ddots & 1 \\
1 & \cdots & 1 & 3 n+2 m+1
\end{array}\right)_{m \times m}\right)^{3} \\
& \times\left(\operatorname{det}\left(\begin{array}{cccc}
3 m+2 n+1 & 1 & \cdots & 1 \\
1 & \ddots & \ddots & \vdots \\
\vdots & \ddots & \ddots & 1 \\
1 & \cdots & 1 & 3 m+2 n+1
\end{array}\right)\right)_{n \times n}
\end{aligned}
$$


Using Lemma 2, we have

$$
\tau\left(K_{3}\left[K_{m, n}\right]\right)=3^{4}(m+n)^{4}(3 m+2 n)^{3 \mathbf{n}-3}(3 n+2 m)^{3 \mathbf{m}-3} .
$$

In particular,

$$
\tau\left(K_{3}\left[K_{n, n}\right]\right)=6^{4} \times 5^{6 n-6} \times n^{6 n-2} ; \quad n \geq 1 .
$$

\section{Complexity of Tensor Product of Graphs}

The tensor product, or Kronecker product, $G_{1} \otimes G_{2}$, is the simple graph with $V\left(G_{1} \otimes G_{2}\right)=V_{1} \times V_{2}$, where $\left(u_{1}, u_{2}\right)$ and $\left(v_{1}, v_{2}\right)$ are adjacent in $G_{1} \otimes G_{2}$ if and only if $u_{1}$ is adjacent to $v_{1}$ in $G_{1}$ and $u_{2}$ is adjacent to $v_{2}$ in $G_{2}$ [13].

Lemma 11. For $m, n \geq 1$, we have

$$
\tau\left(K_{2} \otimes K_{m, n}\right)=0 .
$$

Theorem 12. For $m, n \geq 1$, we have

$$
\begin{aligned}
\tau\left(K_{3} \otimes K_{m, n}\right)= & \times 2^{3 m+3 n-5} \\
& \times n^{3 m-1} \times m^{3 n-1} .
\end{aligned}
$$

Proof. Applying Lemma 1, we have

$$
\begin{aligned}
& \tau\left(K_{3} \otimes K_{m, n}\right) \\
& =\frac{1}{9(m+n)^{2}} \operatorname{det}(3(m+n) I-\bar{D}+\bar{A}) \\
& =\frac{1}{9(m+n)^{2}}
\end{aligned}
$$

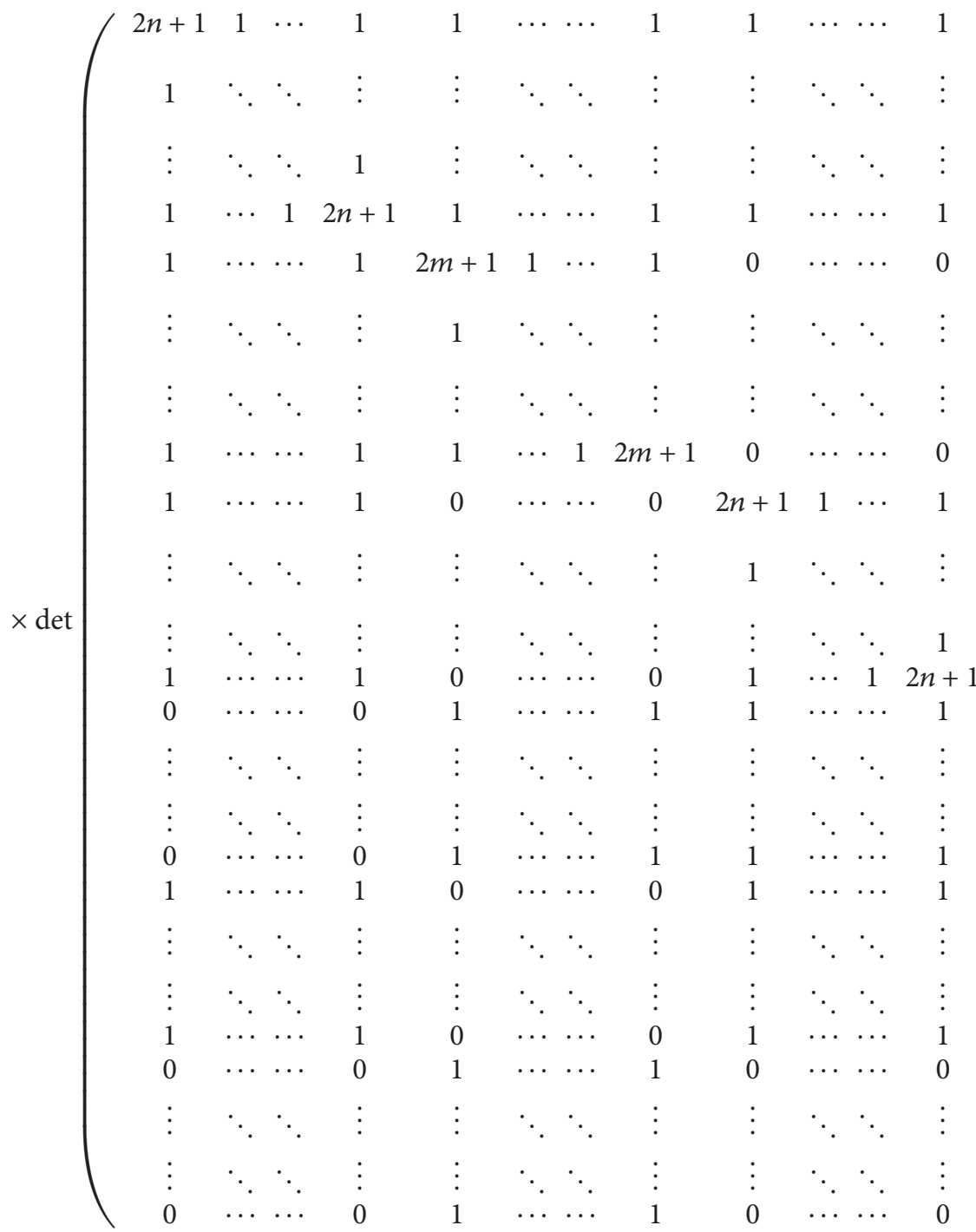




$$
\begin{aligned}
& \begin{array}{cccccccccccc}
0 & \cdots & \cdots & 0 & 1 & \cdots & \cdots & 1 & 0 & \cdots & \cdots & 0 \\
\vdots & \ddots & \ddots & \vdots & \vdots & \ddots & \ddots & \vdots & \vdots & \ddots & \ddots & \vdots \\
\vdots & \ddots & \ddots & \vdots & \vdots & \ddots & \ddots & \vdots & \vdots & \ddots & \ddots & \vdots \\
0 & \cdots & \cdots & 0 & 1 & \cdots & \cdots & 1 & 0 & \cdots & \cdots & 0 \\
1 & \cdots & \cdots & 1 & 0 & \cdots & \cdots & 0 & 1 & \cdots & \cdots & 1
\end{array} \\
& \begin{array}{llllllllllll}
\vdots & \ddots & \ddots & \vdots & \vdots & \ddots & \ddots & \vdots & \vdots & \ddots & \ddots & \vdots
\end{array} \\
& \begin{array}{cccccccccccc}
\vdots & \ddots & \ddots & \vdots & \vdots & \ddots & \ddots & \vdots & \vdots & \ddots & \ddots & \vdots \\
1 & \ldots & \ldots & 1 & 0 & \ldots & \cdots & 0 & 1 & \ldots & \ldots & 1 \\
1 & \ldots & \ldots & 1 & 1 & \ldots & \ldots & 1 & 0 & \ldots & \ldots & 0
\end{array}
\end{aligned}
$$

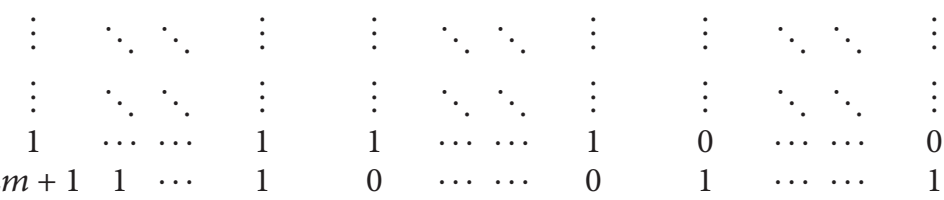

$$
\begin{aligned}
& \begin{array}{llllllllllll}
1 & \ddots & \ddots & \vdots & \vdots & \ddots & \ddots & \vdots & \vdots & \ddots & \ddots & \vdots
\end{array} \\
& \begin{array}{cccccccccccc}
\vdots & \ddots & \ddots & 1 & \vdots & \ddots & \ddots & \vdots & \vdots & \ddots & \ddots & \vdots \\
1 & \cdots & 1 & 2 m+1 & 0 & \cdots & \cdots & 0 & 1 & \cdots & \cdots & 1 \\
0 & \cdots & \cdots & 0 & 2 n+1 & 1 & \cdots & 1 & 1 & \cdots & \cdots & 1
\end{array} \\
& \left.\begin{array}{cccccccccccc}
\vdots & \ddots & \ddots & \vdots & 1 & \ddots & \ddots & \vdots & \vdots & \ddots & \ddots & \vdots \\
\vdots & \ddots & \ddots & \vdots & \vdots & \ddots & \ddots & 1 & \vdots & \ddots & \ddots & \vdots \\
0 & \cdots & \cdots & 0 & 1 & \cdots & 1 & 2 n+1 & 1 & \cdots & \cdots & 1 \\
1 & \cdots & \cdots & 1 & 1 & \cdots & \cdots & 1 & 2 m+1 & 1 & \cdots & 1 \\
\vdots & \ddots & \ddots & \vdots & \vdots & \ddots & \ddots & \vdots & 1 & \ddots & \ddots & \vdots \\
\vdots & \ddots & \ddots & \vdots & \vdots & \ddots & \ddots & \vdots & \vdots & \ddots & \ddots & 1 \\
1 & \cdots & \cdots & 1 & 1 & \cdots & \cdots & 1 & 1 & \cdots & 1 & 2 m+1
\end{array}\right) \\
& =\frac{1}{9(m+n)^{2}} \operatorname{det}\left(\begin{array}{ccc}
A & B & B \\
B & A & B \\
B & B & A
\end{array}\right)=\frac{1}{9(m+n)^{2}}[\operatorname{det}(A-B)]^{2}[\operatorname{det}(A+2 B)] \\
& =\frac{1}{9(m+n)^{2}}\left(\operatorname{det}\left(\begin{array}{cccccccc}
2 n & 0 & \cdots & 0 & 1 & \cdots & \cdots & 1 \\
0 & \ddots & \ddots & \vdots & \vdots & \ddots & \ddots & \vdots \\
\vdots & \ddots & \ddots & 0 & \vdots & \ddots & \ddots & \vdots \\
0 & \cdots & 0 & 2 n & 1 & \cdots & \cdots & 1 \\
1 & \cdots & \cdots & 1 & 2 m & 0 & \cdots & 0 \\
\vdots & \ddots & \ddots & \vdots & 0 & \ddots & \ddots & \vdots \\
\vdots & \ddots & \ddots & \vdots & \vdots & \ddots & \ddots & 0 \\
1 & \cdots & \cdots & 1 & 0 & \cdots & 0 & 2 m
\end{array}\right)\right)^{2} \\
& \times \operatorname{det}\left(\begin{array}{cccccccc}
2 n+3 & 3 & \cdots & 3 & 1 & \cdots & \cdots & 1 \\
3 & \ddots & \ddots & \vdots & \vdots & \ddots & \ddots & \vdots \\
\vdots & \ddots & \ddots & 3 & \vdots & \ddots & \ddots & \vdots \\
3 & \cdots & 3 & 2 n+3 & 1 & \cdots & \cdots & 1 \\
1 & \cdots & \cdots & 1 & 2 m+3 & 3 & \cdots & 3 \\
\vdots & \ddots & \ddots & \vdots & 3 & \ddots & \ddots & \vdots \\
\vdots & \ddots & \ddots & \vdots & \vdots & \ddots & \ddots & 3 \\
1 & \cdots & \cdots & 1 & 3 & \cdots & 3 & 2 m+3
\end{array}\right)
\end{aligned}
$$




$$
\begin{aligned}
= & \frac{1}{9(m+n)^{2}} \operatorname{det}\left(\begin{array}{cc}
A & B \\
B^{T} & C
\end{array}\right) \times \operatorname{det}\left(\begin{array}{cc}
D & E \\
E^{T} & F
\end{array}\right)=\frac{1}{9(m+n)^{2}} \\
& \times(\operatorname{det} A)^{2}\left(\operatorname{det}\left(C-B^{T} A^{-1} B\right)\right)^{2} \times \operatorname{det} D \operatorname{det}\left(F-E^{T} D^{-1} E\right) .
\end{aligned}
$$

Thus,

$$
\begin{aligned}
& \tau\left(K_{3} \otimes K_{m, n}\right)=\frac{1}{9(m+n)^{2}}\left(\operatorname{det}\left(\begin{array}{cccc}
2 n & 0 & \cdots & 0 \\
0 & \ddots & \ddots & \vdots \\
\vdots & \ddots & \ddots & 0 \\
0 & \cdots & 0 & 2 n
\end{array}\right)_{m \times m}\right)^{2} \\
& \times\left(\operatorname{det}\left(\begin{array}{cccc}
\frac{m(4 n-1)}{2 n} & \frac{-m}{2 n} & \cdots & \frac{-m}{2 n} \\
\frac{-m}{2 n} & \ddots & \ddots & \vdots \\
\vdots & \ddots & \ddots & \frac{-m}{2 n} \\
\frac{-m}{2 n} & \cdots & \frac{-m}{2 n} & \frac{m(4 n-1)}{2 n}
\end{array}\right)_{n \times n}\right)^{2} \\
& \times \operatorname{det}\left(\begin{array}{cccc}
2 n+3 & 3 & \cdots & 3 \\
3 & \ddots & \ddots & \vdots \\
\vdots & \ddots & \ddots & 3 \\
3 & \cdots & 3 & 2 n+3
\end{array}\right)_{m \times m} \\
& \times \operatorname{det}\left(\begin{array}{cccc}
\frac{n(4 m+6)+6 m^{2}+8 m}{2 n+3 m} & \frac{6 n+8 m}{2 n+3 m} & \cdots & \frac{6 n+8 m}{2 n+3 m} \\
\frac{6 n+8 m}{2 n+3 m} & \ddots & \ddots & \vdots \\
\vdots & \ddots & \ddots & \frac{6 n+8 m}{2 n+3 m} \\
\frac{6 n+8 m}{2 n+3 m} & \cdots & \frac{6 n+8 m}{2 n+3 m} & \frac{n(4 m+6)+6 m^{2}+8 m}{2 n+3 m}
\end{array}\right)_{n \times n} \\
& =\frac{1}{9(m+n)^{2}}(2 n)^{2 m} \times\left(\frac{-m}{2 n}\right)^{2 n} \times\left(\operatorname{det}\left(\begin{array}{cccc}
\frac{m(4 n-1)}{-m} & 1 & \cdots & 1 \\
1 & \ddots & \ddots & \vdots \\
\vdots & \ddots & \ddots & 1 \\
1 & \cdots & 1 & \frac{m(4 n-1)}{-m}
\end{array}\right)\right)_{n \times n} \\
& \times 3^{m} \times \operatorname{det}\left(\begin{array}{cccc}
\frac{2 n+3}{3} & 1 & \cdots & 1 \\
1 & \ddots & \ddots & \vdots \\
\vdots & \ddots & \ddots & 1 \\
1 & \cdots & 1 & \frac{2 n+3}{3}
\end{array}\right) \times\left(\frac{6 n+8 m}{2 n+3 m}\right)^{n}
\end{aligned}
$$




$$
\times \operatorname{det}\left(\begin{array}{cccc}
\frac{n(4 m+6)+6 m^{2}+8 m}{6 n+8 m} & 1 & \cdots & 1 \\
1 & \ddots & \ddots & \vdots \\
\vdots & \ddots & \ddots & 1 \\
1 & \cdots & 1 & \frac{n(4 m+6)+6 m^{2}+8 m}{6 n+8 m}
\end{array}\right)_{n \times n}
$$

Using Lemma 2, we have

$$
\begin{aligned}
\tau\left(K_{3} \otimes K_{m, n}\right)= & \frac{1}{9(m+n)^{2}}(2 n)^{2 m} \\
& \times\left[\left(\frac{m}{2 n}\right)^{2 n} \times(-3 n)^{2} \times(-4 n)^{2 n-2}\right] \\
& \times\left[3^{m}\left(\frac{2 n+3 m}{3}\right) \times\left(\frac{2 n}{3}\right)^{m-1}\right] \\
& \left.\times\left(\frac{6 n+8 m}{2 n+3 m}\right)^{n}\right] \\
& \times\left[\left(\frac{4 n m+6 n+6 m^{2}+8 m}{6 n+8 m}+n-1\right)\right. \\
& \left.\times\left(\frac{4 n m+6 n+6 m^{2}+8 m}{6 n+8 m^{2}}-1\right)^{n-1}\right] \\
= & 3 \times(2 n)^{2 m-2 n} \times m^{3 n-1} \\
& \times n^{2 n+m-1} \times 2^{5 n+m-5}
\end{aligned}
$$

$$
=3 \times 2^{3 m+3 n-5} \times n^{3 m-1} \times m^{3 n-1} .
$$

In particular,

$$
\tau\left(K_{3} \otimes K_{n, n}\right)=3 \times 2^{6 n-5} \times n^{6 n-2} ; \quad n \geq 1 .
$$

\section{Number of Spanning Trees of Symmetric Product of Graphs}

The symmetric product, $G_{1} \oplus G_{2}$, is the simple graph with $V\left(G_{1} \circ G_{2}\right)=V_{1} \times V_{2}$, where $\left(u_{1}, u_{2}\right)$ and $\left(v_{1}, v_{2}\right)$ are adjacent in $G_{1} \oplus G_{2}$ if and only if either $u_{1}$ is adjacent to $v_{1}$ in $G_{1}$ and $u_{2}$ is not adjacent to $v_{2}$ in $G_{2}$, or $u_{1}$ is not adjacent to $v_{1}$ in $G_{1}$ and $u_{2}$ is adjacent to $v_{2}$ in $G_{2}$ [13].

Theorem 13. For $n, m \geq 1$, we have

$$
\tau\left(K_{2} \oplus K_{m, n}\right)=(m+n)^{2(m+n-1)} .
$$

Proof. Applying Lemma 1, we have

$$
\begin{aligned}
& \tau\left(K_{2} \oplus K_{m, n}\right) \\
& =\frac{1}{4(m+n)^{2}} \operatorname{det}(2(m+n) I-\bar{D}+\bar{A}) \\
& =\frac{1}{4(m+n)^{2}}
\end{aligned}
$$




$$
\times \operatorname{det}\left(\begin{array}{cccccccc}
m+n+1 & 1 & \cdots & 1 & 0 & \cdots & \cdots & 0 \\
1 & \ddots & \ddots & \vdots & \vdots & \ddots & \ddots & \vdots \\
\vdots & \ddots & \ddots & 1 & \vdots & \ddots & \ddots & \vdots \\
1 & \cdots & 1 & m+n+1 & 0 & \cdots & \cdots & 0 \\
0 & \cdots & \cdots & 0 & m+n+1 & 1 & \cdots & 1 \\
\vdots & \ddots & \ddots & \vdots & 1 & \ddots & \ddots & \vdots \\
\vdots & \ddots & \ddots & \vdots & \vdots & \ddots & \ddots & 1 \\
0 & \cdots & \cdots & 0 & 1 & \cdots & 1 & m+n+1 \\
0 & \cdots & \cdots & 0 & 1 & \cdots & \cdots & 1 \\
\vdots & \ddots & \ddots & \vdots & \vdots & \ddots & \ddots & \vdots \\
\vdots & \ddots & \ddots & \vdots & \vdots & \ddots & \ddots & \vdots \\
0 & \cdots & \cdots & 0 & 1 & \cdots & \cdots & 1 \\
1 & \cdots & \cdots & 1 & 0 & \cdots & \cdots & 0 \\
\vdots & \ddots & \ddots & \vdots & \vdots & \ddots & \ddots & \vdots \\
\vdots & \ddots & \ddots & \vdots & \vdots & \ddots & \ddots & \vdots \\
1 & \cdots & \cdots & 1 & 0 & \cdots & \cdots & 0
\end{array}\right.
$$

$$
\begin{aligned}
& \begin{array}{lllll}
0 & \ldots & \ldots & 0 & 1
\end{array}
\end{aligned}
$$

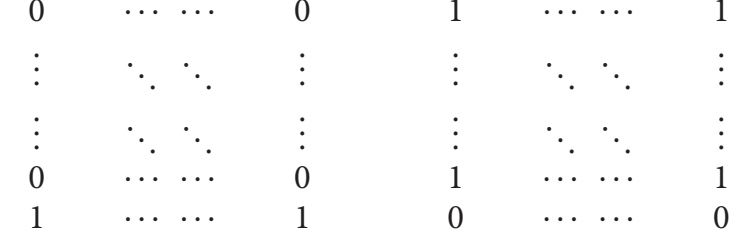

$$
\begin{aligned}
& \begin{array}{llllllll}
\vdots & \ddots & \ddots & \vdots & \vdots & \ddots & \ddots & \vdots
\end{array} \\
& \begin{array}{cccccccc}
\vdots & \ddots & \ddots & \vdots & \vdots & \ddots & \ddots & \vdots \\
1 & \ldots & \cdots & 1 & 0 & \ldots & \cdots & 0
\end{array} \\
& \begin{array}{llllllll}
m+n+1 & 1 & \cdots & 1 & 0 & \ldots & \cdots & 0
\end{array} \\
& \left.\begin{array}{cccccccc}
1 & \ddots & \ddots & \vdots & \vdots & \ddots & \ddots & \vdots \\
\vdots & \ddots & \ddots & 1 & \vdots & \ddots & \ddots & \vdots \\
1 & \cdots & 1 & m+n+1 & 0 & \cdots & \cdots & 0 \\
0 & \ddots & \ddots & 0 & m+n+1 & 1 & \cdots & 1 \\
\vdots & \ddots & \ddots & \vdots & 1 & \ddots & \ddots & \vdots \\
\vdots & \ddots & \ddots & \vdots & \vdots & \ddots & \ddots & 1 \\
0 & \cdots & \cdots & 0 & 1 & \cdots & 1 & m+n+1
\end{array}\right) \\
& =\frac{1}{4(m+n)^{2}} \operatorname{det}\left(\begin{array}{cccccccc}
m+n+1 & 1 & \cdots & 1 & 1 & \cdots & \cdots & 1 \\
1 & \ddots & \ddots & \vdots & \vdots & \ddots & \ddots & \vdots \\
\vdots & \ddots & \ddots & 1 & \vdots & \ddots & \ddots & \vdots \\
1 & \cdots & 1 & m+n+1 & 1 & \cdots & \cdots & 1 \\
1 & \cdots & \cdots & 1 & m+n+1 & 1 & \cdots & 1 \\
\vdots & \ddots & \ddots & \vdots & 1 & \ddots & \ddots & \vdots \\
\vdots & \ddots & \ddots & \vdots & \vdots & \ddots & \ddots & 1 \\
1 & \cdots & \cdots & 1 & 1 & \cdots & 1 & m+n+1
\end{array}\right)
\end{aligned}
$$




$$
\begin{aligned}
& \times \operatorname{det}\left(\begin{array}{cccccccc}
m+n+1 & 1 & \cdots & 1 & -1 & \cdots & \cdots & -1 \\
1 & \ddots & \ddots & \vdots & \vdots & \ddots & \ddots & \vdots \\
\vdots & \ddots & \ddots & 1 & \vdots & \ddots & \ddots & \vdots \\
1 & \cdots & 1 & m+n+1 & -1 & \cdots & \cdots & -1 \\
-1 & \cdots & \cdots & -1 & m+n+1 & 1 & \cdots & 1 \\
\vdots & \ddots & \ddots & \vdots & 1 & \ddots & \ddots & \vdots \\
\vdots & \ddots & \ddots & \vdots & \vdots & \ddots & \ddots & 1 \\
-1 & \cdots & \cdots & -1 & 1 & \cdots & 1 & m+n+1
\end{array}\right) \\
& =\frac{1}{4(m+n)^{2}}(m+n+1+m+n-1)(m+n+1-1)^{m+n-1} \times \operatorname{det}\left(\begin{array}{cc}
A & B \\
B^{T} & C
\end{array}\right) \\
& =\frac{1}{2}(m+n)^{m+n-2} \times \operatorname{det} A \operatorname{det}\left(C-B^{T} A^{-1} B\right) \\
& =\frac{1}{2}(m+n)^{m+n-2} \times(2 m+n)(m+n)^{m-1} \\
& \times \operatorname{det}\left(\begin{array}{cccc}
\frac{n^{2}+(3 m+1) n+2 m^{2}+m}{(n+2 m)} & \frac{n+m}{n+2 m} & \cdots & \frac{n+m}{n+2 m} \\
\frac{n+m}{n+2 m} & \ddots & \ddots & \vdots \\
\vdots & \ddots & \ddots & \frac{n+m}{n+2 m} \\
\frac{n+m}{n+2 m} & \cdots & \frac{n+m}{n+2 m} & \frac{n^{2}+(3 m+1) n+2 m^{2}+m}{(n+2 m)}
\end{array}\right)_{n \times n} \\
& =\frac{1}{2}(m+n)^{2 m+n-3} \times(2 m+n) \times\left(\frac{n+m}{n+2 m}\right)^{n} \\
& \times \operatorname{det}\left(\begin{array}{cccc}
\frac{n^{2}+(3 m+1) n+2 m^{2}+m}{n+m} & 1 & \cdots & 1 \\
1 & \ddots & \ddots & \vdots \\
\vdots & \ddots & \ddots & 1 \\
1 & \cdots & 1 & \frac{n^{2}+(3 m+1) n+2 m^{2}+m}{n+m}
\end{array}\right)_{n \times n}
\end{aligned}
$$

Thus,

$$
\begin{aligned}
\tau\left(K_{2} \oplus K_{m, n}\right)= & \frac{1}{2}(m+n)^{2 m+n-3} \times(2 m+n) \\
& \times\left(\frac{n+m}{n+2 m}\right)^{n} \times(2 n+2 m)(n+2 m)^{n-1} \\
= & (m+n)^{2(m+n-1)} .
\end{aligned}
$$

In particular,

$$
\tau\left(K_{2} \oplus K_{n, n}\right)=(2 n)^{2(2 n-1)} ; \quad n \geq 1 .
$$

Theorem 14. For $m, n \geq 1$, we have

$$
\begin{aligned}
\tau\left(K_{3} \oplus K_{m, n}\right)= & 3(2 m+n)^{3 m-3} \\
& \times(2 n+m)^{3 n-3}\left(m^{2}+n^{2}+3 m n\right)^{2} .
\end{aligned}
$$

Proof. Applying Lemma 1, we have

$$
\begin{aligned}
& \tau\left(K_{3} \oplus K_{m, n}\right) \\
& =\frac{1}{9(m+n)^{2}} \operatorname{det}(3(m+n) I-\bar{D}+\bar{A}) \\
& =\frac{1}{9(m+n)^{2}}
\end{aligned}
$$




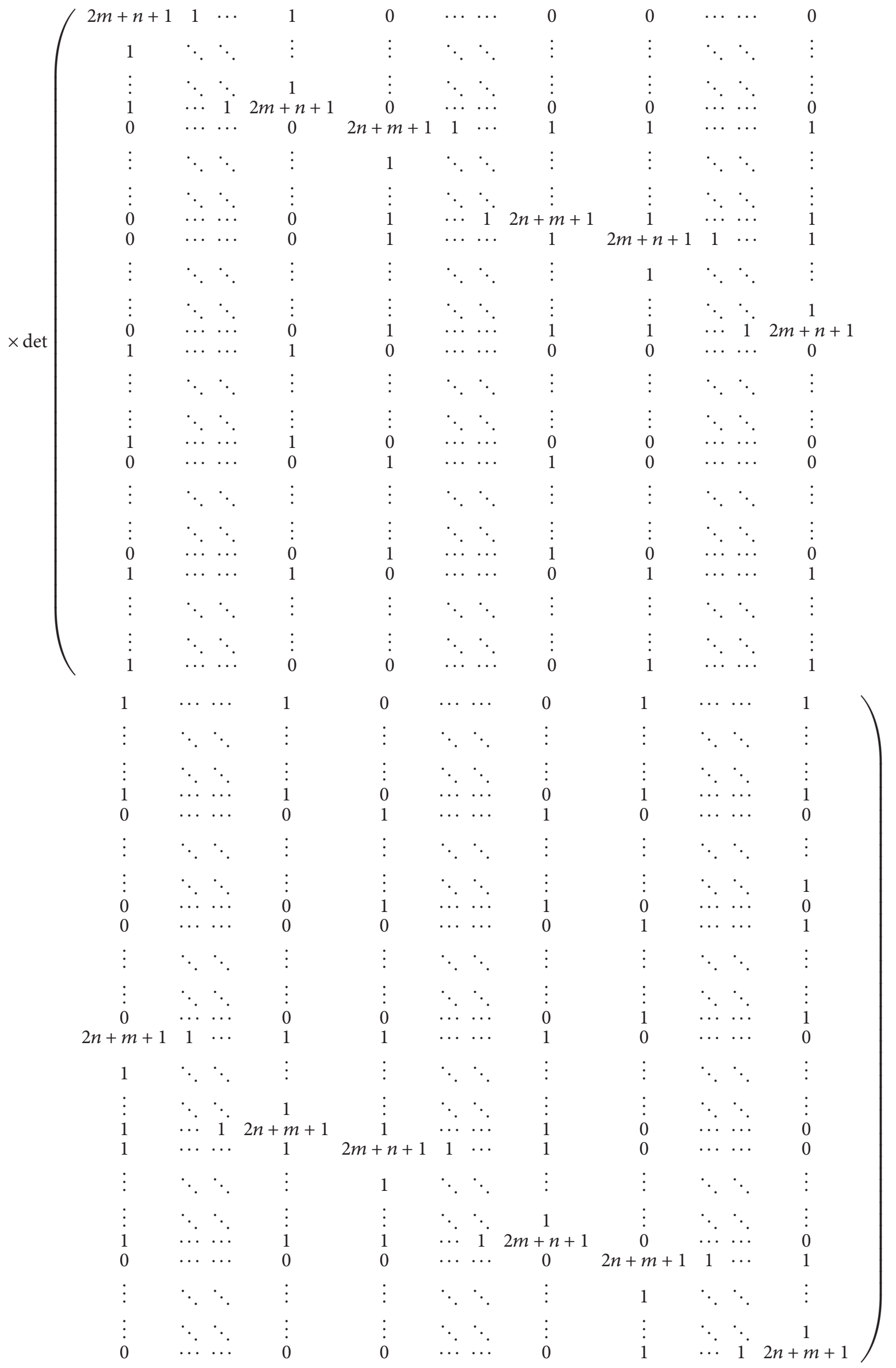




$$
\begin{aligned}
& =\frac{1}{9(m+n)^{2}} \operatorname{det}\left(\begin{array}{ccc}
A & B & B \\
B & A & B \\
B & B & A
\end{array}\right)=\frac{1}{9(m+n)^{2}}[\operatorname{det}(A-B)]^{2}[\operatorname{det}(A+2 B)] \\
& =\frac{1}{9(m+n)^{2}}\left(\operatorname{det}\left(\begin{array}{cccccccc}
2 m+n+1 & 1 & \cdots & 1 & -1 & \cdots & \cdots & -1 \\
1 & \ddots & \ddots & \vdots & \vdots & \ddots & \ddots & \vdots \\
\vdots & \ddots & \ddots & 1 & \vdots & \ddots & \ddots & \vdots \\
1 & \cdots & 1 & 2 m+n+1 & -1 & \cdots & \cdots & -1 \\
-1 & \cdots & \cdots & -1 & 2 n+m+1 & 1 & \cdots & 1 \\
\vdots & \ddots & \ddots & \vdots & 1 & \ddots & \ddots & \vdots \\
\vdots & \ddots & \ddots & \vdots & \vdots & \ddots & \ddots & 1 \\
-1 & \cdots & \cdots & -1 & 1 & \cdots & 1 & 2 n+m+1
\end{array}\right)^{2}\right. \\
& \times \operatorname{det}\left(\begin{array}{cccccccc}
2 m+n+1 & 1 & \cdots & 1 & 2 & \cdots & \cdots & 2 \\
1 & \ddots & \ddots & \vdots & \vdots & \ddots & \ddots & \vdots \\
\vdots & \ddots & \ddots & 1 & \vdots & \ddots & \ddots & \vdots \\
1 & \cdots & 1 & 2 m+n+1 & 2 & \cdots & \cdots & 2 \\
2 & \cdots & \cdots & 2 & 2 n+m+1 & 1 & \cdots & 1 \\
\vdots & \ddots & \ddots & \vdots & 3 & \ddots & \ddots & \vdots \\
\vdots & \ddots & \ddots & \vdots & \vdots & \ddots & \ddots & 1 \\
2 & \cdots & \cdots & 2 & 1 & \cdots & 1 & 2 n+m+1
\end{array}\right) \\
& =\frac{1}{9(m+n)^{2}}\left(\operatorname{det}\left(\begin{array}{cc}
A & B \\
B^{T} & C
\end{array}\right)\right)^{2} \times \operatorname{det}\left(\begin{array}{cc}
D & E \\
E^{T} & F
\end{array}\right)=\frac{1}{9(m+n)^{2}} \times(\operatorname{det} A)^{2}\left(\operatorname{det}\left(C-B^{T} A^{-1} B\right)\right)^{2} \\
& \times \operatorname{det} D \operatorname{det}\left(F-E^{T} D^{-1} E\right) \\
& =\frac{(3 m+n)^{2}(2 m+n)^{2 m-2}}{9(m+n)^{2}}
\end{aligned}
$$$$
\times\left(\operatorname{det}\left(\begin{array}{cccc}
\frac{2 n^{2}+n(7 m+1)+3 m^{2}+2 m}{n+3 m} & \frac{n+2 m}{n+3 m} & \cdots & \frac{n+2 m}{n+3 m} \\
\frac{n+2 m}{n+3 m} & \ddots & \ddots & \vdots \\
\vdots & \ddots & \ddots & \frac{n+2 m}{n+3 m} \\
\frac{n+2 m}{n+3 m} & \cdots & \frac{n+2 m}{n+3 m} & \frac{2 n^{2}+n(7 m+1)+3 m^{2}+2 m}{n+3 m}
\end{array}\right)_{n \times n}^{n}\right.
$$$$
\times(3 m+n)(2 m+n)^{m-1}
$$$$
\times \operatorname{det}\left(\begin{array}{cccc}
\frac{2 n^{2}+n(7 m+1)+3 m^{2}-m}{n+3 m} & \frac{n-m}{n+3 m} & \cdots & \frac{n-m}{n+3 m} \\
\frac{n-m}{n+3 m} & \ddots & \ddots & \vdots \\
\vdots & \ddots & \ddots & \frac{n-m}{n+3 m} \\
\frac{n-m}{n+3 m} & \cdots & \frac{n-m}{n+3 m} & \frac{2 n^{2}+n(7 m+1)+3 m^{2}-m}{n+3 m}
\end{array}\right)_{n \times n}
$$ 


$$
\begin{aligned}
= & \frac{(3 m+n)^{2}(2 m+n)^{2 m-2}}{9(m+n)^{2}} \\
& \left.\times\left(\frac{n+2 m}{n+3 m}\right)^{2 n} \operatorname{det}\left(\begin{array}{cccc}
\frac{2 n^{2}+n(7 m+1)+3 m^{2}+2 m}{n+2 m} & 1 & \cdots & 1 \\
1 & \ddots & \ddots & \vdots \\
\vdots & \ddots & \ddots & 1 \\
1 & \cdots & 1 & \left.\frac{2 n^{2}+n(7 m+1)+3 m^{2}+2 m}{n+2 m}\right)_{n \times n}
\end{array}\right)\right)^{2} \\
& \times(3 m+n)(2 m+n)^{m-1}
\end{aligned}
$$$$
\times\left(\frac{n-m}{n+3 m}\right)^{n} \operatorname{det}\left(\begin{array}{cccc}
\frac{2 n^{2}+n(7 m+1)+3 m^{2}-m}{n-m} & 1 & \cdots & 1 \\
1 & \ddots & \ddots & \vdots \\
\vdots & \ddots & \ddots & 1 \\
1 & \cdots & 1 & \frac{2 n^{2}+n(7 m+1)+3 m^{2}-m}{n-m}
\end{array}\right)_{n \times n}
$$

Using Lemma 2, we have

$$
\begin{aligned}
\tau\left(K_{3} \oplus K_{m, n}\right)= & \frac{1}{9(m+n)^{2}}(3 m+n)^{2}(2 m+n)^{2 m-2} \\
& \times\left(\frac{n+2 m}{n+3 m}\right)^{2 n} \frac{1}{(n+3 m)^{2 n}} \\
& \times\left(3 n^{2}+3 m^{2}+9 n m\right)^{2} \\
& \times\left(2 n^{2}+3 m^{2}+7 n m\right)^{2 n-2} \\
& \times(3 m+n)(2 m+n)^{m-1} \\
& \times\left(\frac{n-m}{n+3 m}\right)^{n} \times \frac{1}{(n-m)^{n}} \\
& \times\left(3 n^{2}+3 m^{2}+6 n m\right) \\
& \times\left(2 n^{2}+3 m^{2}+7 n m\right)^{n-1} \\
= & 3(2 m+n)^{3 m-3}(2 n+m)^{3 n-3} \\
& \times\left(m^{2}+n^{2}+3 m n\right)^{2} . \\
&
\end{aligned}
$$

In particular,

$$
\tau\left(K_{3} \oplus K_{n, n}\right)=25 \times 3^{6 n-5} \times n^{6 n-2} ; \quad n \geq 1 .
$$

\section{Conclusion}

The number of spanning trees $\tau(G)$ in graphs (networks) is an important invariant. The evaluation of this number is not only interesting from a mathematical (computational) perspective but is also an important measure of reliability of a network and designing electrical circuits. Some computationally hard problems such as the travelling salesman problem can be solved approximately by using spanning trees. Due to the high dependence of the network design and reliability on the graph theory, we introduced the above important theorems and lemmas and their proofs.

\section{Acknowledgments}

The author is deeply indebted and thankful to the deanship of the scientific research for its help and to the distinct team of employees at Taibah University, Al Madinah Saudi Arabia. This research work was supported by Grant No. 3070/1434.

\section{References}

[1] G. G. Kirchhoff, "Uber die Auflosung der Gleichungen, auf welche man be ider Untersuchung der Linearen Verteilung galvanischer Storme gefuhrt wird," Annual Review of Physical Chemistry, vol. 72, pp. 497-4508, 1847.

[2] A. K. Kelmans and V. M. Chelnokov, "A certain polynomial of a graph and graphs with an extremal number of trees," Journal of Combinatorial Theory B, vol. 16, pp. 197-214, 1974.

[3] G. A. Cayley, "A theorm on trees," Quarterly Journal of Mathematics, vol. 23, pp. 276-378, 1889.

[4] L. Clark, "On the enumeration of spanning trees of the complete multipartite graph," Bulletin of the Institute of Combinatorics and its Applications, vol. 38, pp. 50-60, 2003.

[5] S. N. Qiao and B. Chen, "The number of spanning trees and chains of graphs," Applied Mathematics E-Notes, vol. 9, pp. 10$16,2009$. 
[6] J. Sedláček, "Lucas numbers in graph theory," in Mathematics (Geometry and Graph Theory), pp. 111-115, Univerzita Karlova, Prague, Czech Republic, 1970.

[7] J. Sedlacek, "On the skeleton of a graph or digraph," in Combinatorial Structures and Their Applications, R. Guy, M. Hanani, N. Saver, and J. Schonheim, Eds., pp. 387-391, Gordon and Breach, New York, NY, USA, 1970.

[8] F. T. Boesch and Z. R. Bogdanowicz, "The number of spanning trees in a Prism," International Journal of Computer Mathematics, vol. 21, pp. 229-243, 1987.

[9] F. T. Boesch and H. Prodinger, "Spanning tree formulas and Chebyshev polynomials," Graphs and Combinatorics, vol. 2, no. 3, pp. 191-200, 1986.

[10] S. N. Daoud, "Chebyshev polynomials and spanning tree formulas," International Journal of Mathematical Combinatorics, vol. 4, pp. 68-79, 2013.

[11] M. Marcus, A Servy of Matrix Theory and Matrix Inequalities, Allyn and Bacon, Boston, Mass, USA, 1964.

[12] J. L. Gross and T. W. Tucker, Topological Graph Theory, John Wiley \& Sons, New York, Ny, USA, 1987.

[13] R. Balakrishnan and K. Ranganathan, A textbook of Graph Theory, Springer, New York, NY, USA, 2000. 


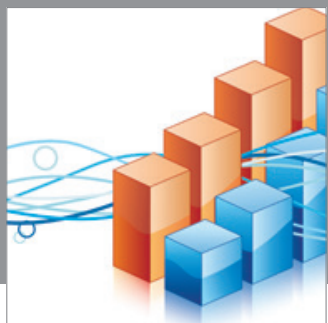

Advances in

Operations Research

mansans

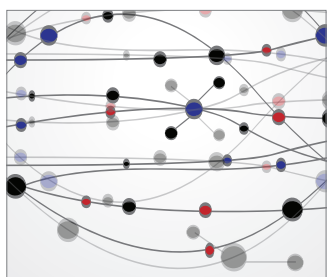

The Scientific World Journal
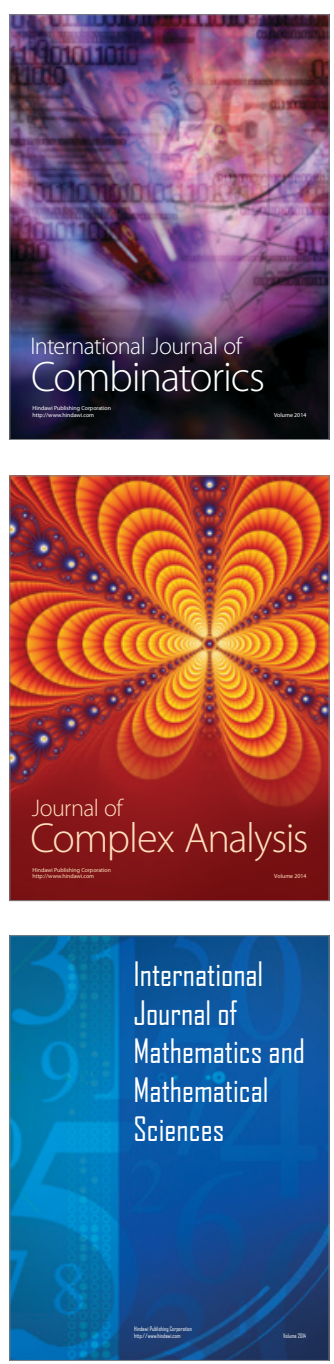
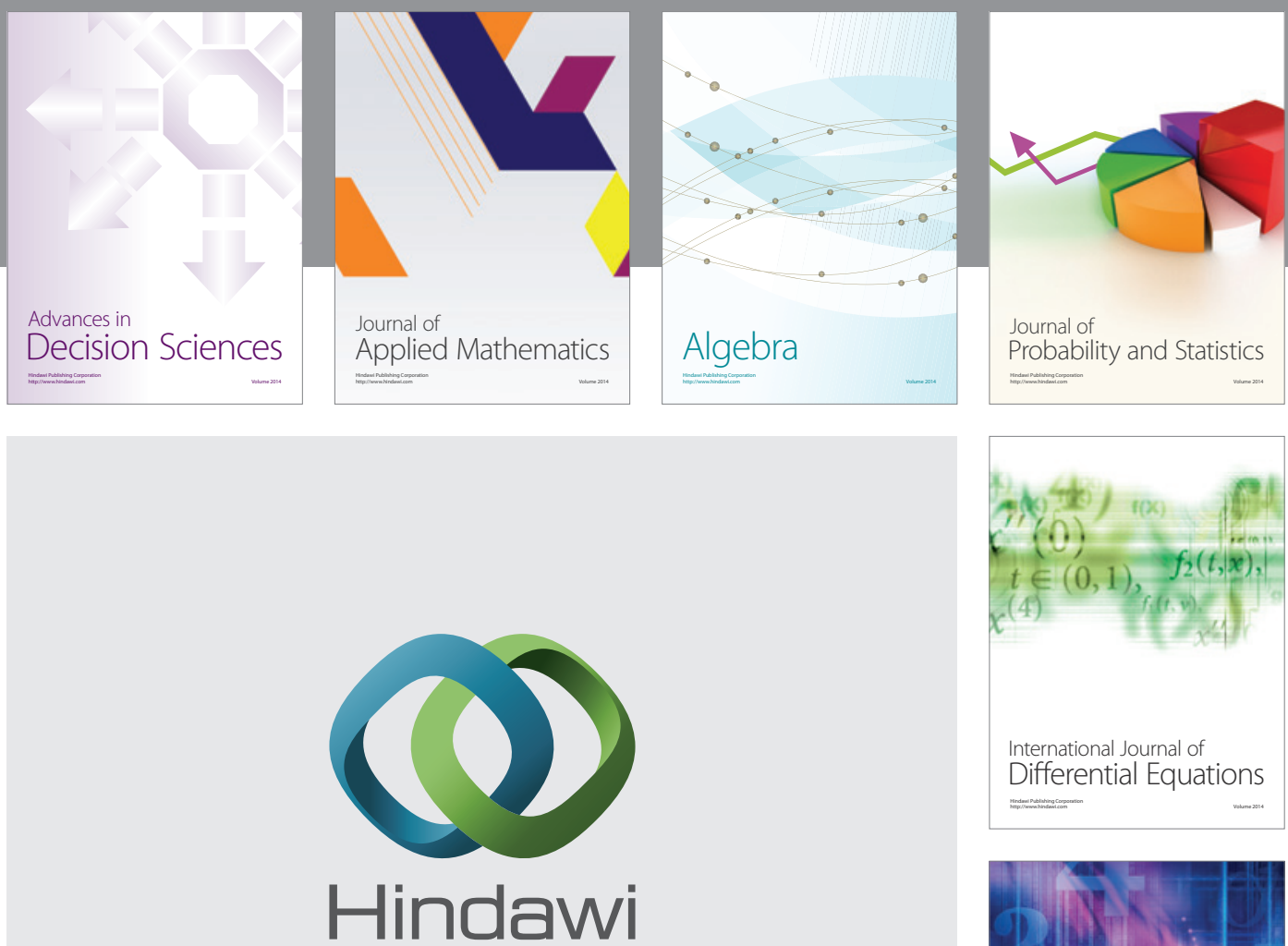

Submit your manuscripts at http://www.hindawi.com
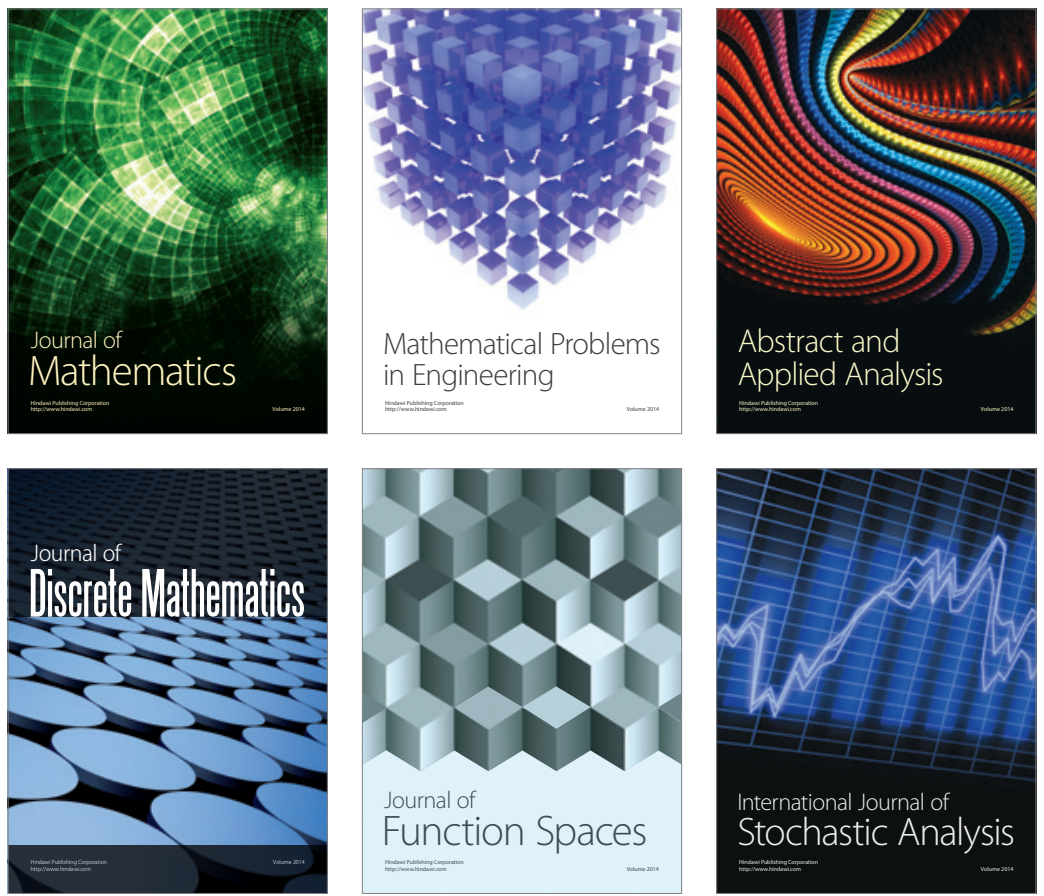

Journal of

Function Spaces

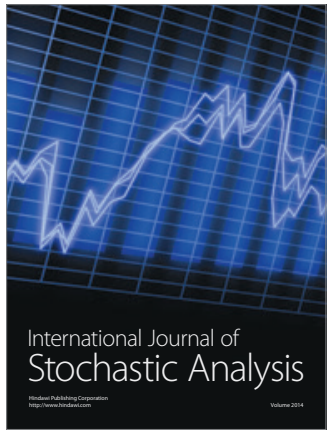

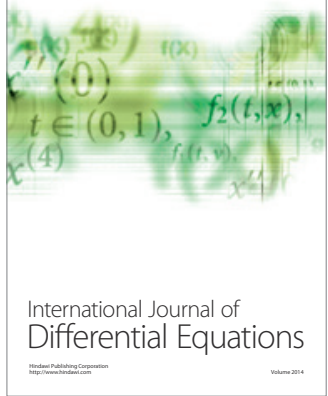
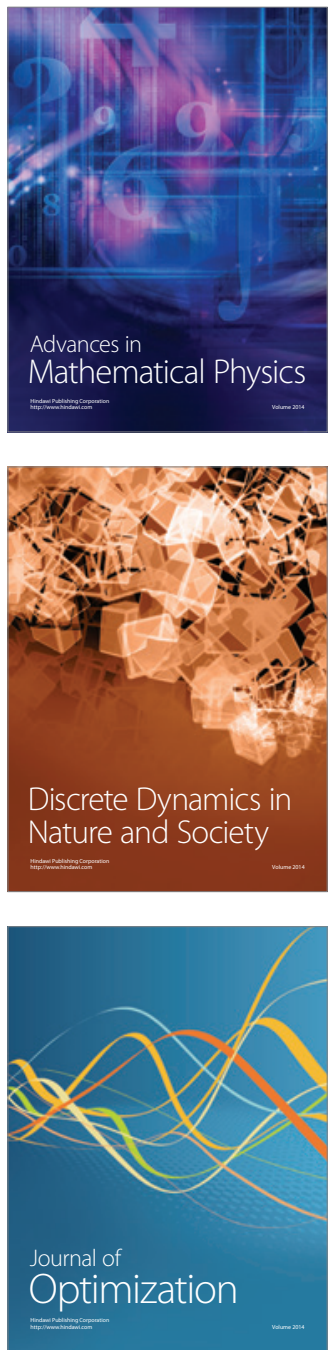